Topological Methods in Nonlinear Analysis

Volume 48, No. 1, 2016, 71-101

DOI: 10.12775 /TMNA. 2016.040

(C) 2016 Juliusz Schauder Centre for Nonlinear Studies

Nicolaus Copernicus University

\title{
MULTI-BUMP SOLUTIONS \\ FOR A CLASS OF KIRCHHOFF TYPE PROBLEMS WITH CRITICAL GROWTH IN $\mathbb{R}^{N}$
}

\author{
SiHUA Liang - Jihui Zhang
}

ABSTRACT. Using variational methods, we establish existence of multibump solutions for a class of Kirchhoff type problems

$$
-\left(1+b \int_{\mathbb{R}^{N}}|\nabla u|^{p} d x\right) \Delta_{p} u+(\lambda V(x)+Z(x)) u^{p-1}=\alpha f(u)+u^{p^{*}-1},
$$

where $f$ is a continuous function, $V, Z: \mathbb{R}^{N} \rightarrow \mathbb{R}$ are continuous functions verifying some hypotheses. We show that if the zero set of $V$ has several isolated connected components $\Omega_{1}, \ldots, \Omega_{k}$ such that the interior of $\Omega_{i}$ is not empty and $\partial \Omega_{i}$ is smooth, then for $\lambda>0$ large enough there exists, for any non-empty subset $\Gamma \subset\{1, \ldots, k\}$, a bump solution trapped in a neighbourhood of $\bigcup_{j \in \Gamma} \Omega_{j}$. The results are also new for the case $p=2$.

2010 Mathematics Subject Classification. 35J60, 35Q55.

Key words and phrases. Kirchhoff type problems; potential well; multi-bump solutions; variational methods.

The first author is supported by the National Natural Science Foundation of China (Grant No. 11301038), The Natural Science Foundation of Jilin Province (Grant No. 20160101244JC), The open project program of Key Laboratory of Symbolic Computation and Knowledge Engineering of Ministry of Education, Jilin University (Grant no. 93K172013K03).

The second author is supported by the National Natural Science Foundation of China (Grant No. 11571176). 


\section{Introduction}

In this paper, we are concerned with the existence of multi-bump solutions of Kirchhoff type problems

$$
\left\{\begin{array}{l}
-\left(1+b \int_{\mathbb{R}^{N}} \frac{1}{p}|\nabla u|^{p} d x\right) \Delta_{p} u+(\lambda V(x)+Z(x)) u^{p-1}=\alpha f(u)+u^{p^{*}-1} \\
u \in W^{1, p}\left(\mathbb{R}^{N}\right), \quad u>0,
\end{array}\right.
$$

where $\Delta_{p} u:=\operatorname{div}\left(|\nabla u|^{p-2} \nabla u\right)$ is the $p$-Laplacian operator, $p^{*}=N p /(N-p)$ is the so-called Sobolev critical exponent, $2<p<N, N \geq 3, b$ is a positive constant, $\lambda>0$ is a real parameter and $f$ is a continuous function, $V, Z: \mathbb{R}^{N} \rightarrow \mathbb{R}$ are continuous functions with $V(x) \geq 0$ for all $x \in \mathbb{R}^{N}, \Omega=\operatorname{int} V^{-1}(0)$ has $k$ connected components denoted by $\Omega_{j}, j \in\{1, \ldots, k\}, V^{-1}(\{0\})=\bar{\Omega}$ and $\partial \Omega$ is smooth.

For the special case of problems (1.1), i.e. without $(\lambda V(x)+Z(x)) u$, problem (1.1) reduces to the following Dirichlet problem of Kirchhoff type:

$$
\left\{\begin{array}{l}
-\left(1+b \int_{\Omega}|\nabla u|^{2} d x\right) \Delta u=h(u), \quad x \in \Omega \\
\left.u\right|_{\partial \Omega}=0
\end{array}\right.
$$

where $\Omega \subset \mathbb{R}^{N}$ is a smooth bounded domain. Such problems are often referred to as being nonlocal because of the presence of the term $\int_{\Omega}|\nabla u|^{2} d x \Delta u$ which implies that the equation in (1.2) is no longer a pointwise identity. This phenomenon provokes some mathematical difficulties, which make the study of such a class of problems particularly interesting. On the other hand, we have its physical motivation. Indeed, this problem is a generalization of a model introduced by Kirchhoff [32]. More precisely, Kirchhoff proposed a model given by the equation

$$
\rho \frac{\partial^{2} u}{\partial t^{2}}-\left(\frac{\rho_{0}}{h}+\frac{E}{2 L} \int_{0}^{L}\left|\frac{\partial u}{\partial x}\right|^{2} d x\right) \frac{\partial^{2} u}{\partial x^{2}}=0,
$$

where $\rho, \rho_{0}, h, E, L$ are constants, which extends the classical D'Alembert's wave equation, by considering the effects of changes in the length of strings during the vibrations. Equation (1.2) is related to the stationary analogue of problem (1.3). It received much attention only after Lions [33] proposed an abstract framework to the problem. Some important and interesting results can be found, for example, in [9], [16], [19], [20], [29], [37], [41]. In [9], Arosio and Panizzi studied the Cauchy-Dirichlet type problem related to (1.3) in the Hadamard sense as a special case of an abstract second-order Cauchy problem in a Hilbert space. Ma and Rivera [37] obtained positive solutions of such problems by using variational methods. Perera and Zhang [41] obtained a nontrivial solution of (1.2) via 
the Yang index and critical group. He and Zou [29] obtained infinitely many solutions by using the local minimum method and the fountain theorem. Recently, the paper [16] considered (1.2) with concave and convex nonlinearities by using the Nehari manifold and fibering map methods, and obtained the existence of multiple positive solutions.

For the case $p=2$ and $b=0$, problem (1.1) can be rewritten as follows:

$$
\left\{\begin{array}{l}
-\Delta u+(\lambda V(x)+Z(x)) u=h(u), \quad x \in \mathbb{R}^{N}, \\
u \in H^{1}\left(\mathbb{R}^{N}\right), \quad u>0 .
\end{array}\right.
$$

Different approaches have been taken to attack this problem under various hypotheses on the potential and the nonlinearity. For example, in the case when the potential function $\lambda V+Z$ is coercive, Miyagaki [38] proved some existence results for a positive solution to (1.4). For the case when the function $\lambda V+Z$ is 1-periodic, Alves et al. [2] showed the existence of positive solutions to (1.4). If $\lambda V+Z$ is radial, Alves et al. [3] also established the existence of a positive solution to (1.4). The papers cited above proved only the existence of positive solutions; the multiplicity of solutions was established in [10]-[12], [14], [15], [21], [22], [27], [28], [42], [43]. In the case when nonlinearities are assumed to be subcritical, there have been enormous investigations on problem (1.4). In [28], by using a Lyapunov-Schmidt reduction, Floer and Weinstein established the existence of a standing wave solutions of (1.4). Moreover they showed that $u$ concentrates near the given non-degenerate critical point of the potential function. Their methods and results were later generalized by $\mathrm{Oh}$ [40] to the higherdimensional case and the existence of multi-bump solutions concentrating near several non-degenerate critical points of the potential function was obtained. For more results, we refer to Ambrosetti, Badiale and Cingolani [7], Ambrosetti, Malchiodi and Secchi [8], Cingolani and Lazzo [17], Cingolani and Nolasco [18], Del Pino and Felmer [21], [22], Ding and Lin [24], Ding and Wei [25] and the references therein.

Recently, He and Zou [30] considered the following equation:

$$
\left\{\begin{array}{l}
-\left(\varepsilon^{2} a+b \varepsilon \int_{\mathbb{R}^{3}}|\nabla u|^{2} d x\right) \Delta u+V(x) u=f(u), \quad x \in \mathbb{R}^{3}, \\
u \in H^{1}\left(\mathbb{R}^{3}\right), \quad u>0
\end{array}\right.
$$

where $f$ is a $C^{1}$ and subcritical function. By using the Ljusternik-Schnirelmann theory (see [45]) and minimax methods, the author obtained the multiplicity of positive solutions, which concentrate on the minima of $V$ as $\varepsilon \rightarrow 0$. This phenomenon of concentration is very interesting for both mathematicians and physicists. For the nonlinearity with critical growth, Wang, Tian, Xu and Zhang [44] obtained the multiplicity and concentration of positive solutions by using the minimax theorems and the Lusternik-Schnirelmann theory. 
In [26], the authors studied problem (1.1) with $p=2, b=0$, Ding and Tanaka considered problem (1.1) without the critical term and assumed that $\alpha=1$. Supposing that $\Omega$ has $k$ connected components, the authors showed that, for this case, problem (1.1) has at least $2^{k}-1$ solutions, for large enough $\lambda$, establishing the existence of solutions called multi-bumps. The same type of problems were considered by Alves et al. [6], [4] with critical growth respectively. Liang and Shi [36] showed the existence of multi-bump solutions for a class of Kirchhoff type problems by using variational methods.

In the present paper, we show the existence of multi-bump solutions to a class of Kirchhoff type problems (1.1) for the general case $p \geq 2$. We mainly follow the idea of [26], [30]. However, here we use a different approach in some estimates, because the $p$-Laplacian is not linear, and some properties that occur for the 2-Laplacian (Laplacian operator), in our opinion, not necessarily hold for the general case, $p \geq 2$, therefore, a careful analysis is needed. Moreover, our nonlinearity with critical growth and some arguments developed in [26] cannot be applied. So, we modify the sets that appear in the minimax arguments explored in [26]. The arguments developed in this paper are variational, and our main result completes the study made in [26], in the sense that we are working with the $p$-Laplacian and a general class of nonlinearities. As we shall see in the present paper, problem (1.1) can be viewed as a Schrödinger equation coupled with a nonlocal term. The competing effect of the nonlocal term with the critical nonlinearity and the lack of compactness of the embedding of $W^{1, p}\left(\mathbb{R}^{N}\right)$ into the space $L^{p^{*}}\left(\mathbb{R}^{N}\right)$ prevent us from using the variational methods in a standard way. Some new estimates for such Kirchhoff equation involving Palais-Smale sequences, which are key points to apply this kind of theory, should be reestablished. The Moser iterative method [39] has to be applied trickly. Let us point out that although the idea was used before for other problems, the adaptation to the procedure for our problem is not trivial, due to the appearance of the nonlocal term we must reconsider this problem and need more delicate estimates.

We make the following assumptions on $V, Z$ and $f$ throughout this paper:

$\left(\mathrm{V}_{1}\right) V \in C\left(\mathbb{R}^{N}, \mathbb{R}\right)$ satisfies $V(x) \geq 0$.

$\left(\mathrm{V}_{2}\right)$ The potential well $\Omega:=\operatorname{int} V^{-1}(0)$ is a non-empty bounded open set with smooth boundary $\partial \Omega$ and $V^{-1}(0)=\bar{\Omega}$.

$\left(\mathrm{V}_{3}\right)$ There exist two positive constants $M_{0}$ and $M_{1}$ such that the functions $V$ and $Z$ verify

$$
\begin{aligned}
0<M_{0} & \leq V(x)+Z(x) & & \text { for all } x \in \mathbb{R}^{N} \\
|Z(x)| & \leq M_{1} & & \text { for all } x \in \mathbb{R}^{N} .
\end{aligned}
$$

$\left(\mathrm{V}_{4}\right)$ The set $\Omega$ consists of $k$ connected components denoted by $\Omega_{j}, j \in$ $\{1, \ldots, k\}$, satisfying $\operatorname{dist}\left(\Omega_{i}, \Omega_{j}\right)>0$ for $i \neq j$, that is $\Omega=\Omega_{1} \cup \ldots \cup \Omega_{k}$. 
$\left(\mathrm{f}_{1}\right)$ There exists $p<q<p^{*}$ such that

$$
\lim _{|t| \rightarrow \infty} \sup \frac{f(t)}{|t|^{q-1}}=m<+\infty .
$$

$\left(\mathrm{f}_{2}\right) \lim _{s \rightarrow 0} f(s) / s^{p-1}=0$.

$\left(\mathrm{f}_{3}\right)$ There is $2 p<\theta<p^{*}$ verifying $0<\theta F(s) \leq s f(s)$, for all $s \geq 0$, where $F(t)=\int_{0}^{t} f(\tau) d \tau$.

$\left(\mathrm{f}_{4}\right)$ The function $f(t) / t^{p-1}$ is increasing for $t \in[0,+\infty)$.

$\left(\mathrm{f}_{5}\right)$ There are $\varsigma>0$ and $\iota \in\left(2 p, p^{*}\right)$ such that $h(s) \geq \varsigma s^{\iota-1}$ for all $s>0$.

Our main results are the following.

Theorem 1.1. Assume that $\left(\mathrm{V}_{1}\right)-\left(\mathrm{V}_{4}\right)$ and $\left(\mathrm{f}_{1}\right)-\left(\mathrm{f}_{5}\right)$ hold. Then, for any non-empty subset $\Gamma$ of $\{1, \ldots, k\}$, there exist constants $\alpha^{*}>0$ and $\lambda^{*}=\lambda^{*}\left(\alpha^{*}\right)$ such that, for all $\alpha \geq \alpha^{*}$ and $\lambda \geq \lambda^{*}$, problem (1.1) has a family $\left(u_{\lambda}\right)$ of positive solutions which dependence on $\alpha$ verifies: for any sequence $\lambda_{n} \rightarrow \infty$, we can extract a subsequence $\lambda_{n_{i}}$ such that $u_{\lambda_{n_{i}}}$ converges strongly in $W^{1, p}\left(\mathbb{R}^{N}\right)$ to a function $u$ which satisfies $u(x)=0$ for $x \notin \Omega_{\Gamma}$ and the restriction $\left.u\right|_{\Omega_{j}}$ is a least energy solution of

$$
\begin{cases}-\left(1+b \int_{\mathbb{R}^{N}} \frac{1}{p}|\nabla u|^{p} d x\right) \Delta_{p} u+Z(x) u^{p-1}=\alpha f(u)+u^{p^{*}-1}, & x \in \Omega_{j}, j \in \Gamma, \\ u>0, & x \in \Omega_{j}, \\ \left.u\right|_{\partial \Omega}=0, & \end{cases}
$$

where $\Omega_{\Gamma}=\bigcup_{j \in \Gamma} \Omega_{j}$.

THEOREM 1.2. Under the assumptions of Theorem 1.1, there exist constants $\alpha^{*}>0$ and $\lambda^{*}=\lambda^{*}\left(\alpha^{*}\right)$ such that, for all $\alpha \geq \alpha^{*}$ and $\lambda \geq \lambda^{*}$, problem (1.1) has at least $2^{k}-1$ positive solutions.

\section{Main results}

In this section, we outline the variational framework for problem (1.1) and give some preliminary lemmas. Let us define the space of functions

$$
E_{\lambda}:=\left\{u \in W^{1, p}\left(\mathbb{R}^{N}\right): \int_{\mathbb{R}^{N}} V(x)|u|^{p} d x<\infty\right\}
$$

endowed with the norm

$$
\|u\|_{\lambda}=\left(\int_{\mathbb{R}^{N}}\left(|\nabla u|^{p}+(\lambda V(x)+Z(x))|u|^{p}\right) d x\right)^{1 / p} .
$$

For $\lambda \geq 1$ it easy to see that $\left(E_{\lambda},\|\cdot\|\right)$ is a Banach space and we have the following continuous imbedding: $E_{\lambda} \hookrightarrow W^{1, p}\left(\mathbb{R}^{N}\right)$.

We say that $u \in E_{\lambda}$ is a weak solution of (1.1) if, for $v \in E_{\lambda}$, 


$$
\begin{aligned}
& \int_{\mathbb{R}^{N}}|\nabla u|^{p-2} \nabla u \nabla v d x+b\left(\int_{\mathbb{R}^{N}} \frac{1}{p}|\nabla u|^{p} d x\right)\left(\int_{\mathbb{R}^{N}}|\nabla u|^{p-2} \nabla u \nabla v d x\right) \\
& \quad+\int_{\mathbb{R}^{N}}(\lambda V(x)+Z(x))|u|^{p-2} u v d x=\alpha \int_{\mathbb{R}^{N}} f(u) v d x+\int_{\mathbb{R}^{N}} u^{p^{*}-2} u v d x .
\end{aligned}
$$

The nonnegative weak solutions of problem (1.1) are the critical points of $I: E_{\lambda} \rightarrow \mathbb{R}$ given by

$$
\begin{aligned}
I(u):=\frac{1}{p} \int_{\mathbb{R}^{N}}\left(|\nabla u|^{p}+(\lambda V(x)+Z(x))|u|^{p}\right) d x \\
\quad+\frac{b}{2}\left(\int_{\mathbb{R}^{N}} \frac{1}{p}|\nabla u|^{p} d x\right)^{2}-\alpha \int_{\mathbb{R}^{N}} F\left(u_{+}\right) d x-\frac{1}{p^{*}} \int_{\mathbb{R}^{N}} u_{+}^{p^{*}} d x,
\end{aligned}
$$

where $F(s)=\int_{0}^{s} f(\tau) d \tau$ and $u_{+}(x)=\max \{u(x), 0\}$.

Moreover, let us assume that the set $\Omega$ consists of $k$ connected components denoted by $\Omega_{j}, j \in\{1, \ldots, k\}$, satisfying $\operatorname{dist}\left(\Omega_{i}, \Omega_{j}\right)>0$ for $i \neq j$, that is $\Omega=\Omega_{1} \cup \ldots \cup \Omega_{k}$.

We also write for an open set $K \subset \mathbb{R}^{N}$,

$$
E_{\lambda}(K):=\left\{u \in W^{1, p}(K): \int_{K} V(x)|u|^{p} d x<\infty\right\}
$$

and the norms

$$
\|u\|_{\lambda}=\left(\int_{K}\left(|\nabla u|^{p}+(\lambda V(x)+Z(x))|u|^{p}\right) d x\right)^{1 / p} .
$$

In view of $\left(\mathrm{V}_{3}\right)$, we have

$$
M_{0}|u|_{p, K}^{p} \leq \int_{K}\left(|\nabla u|^{p}+(\lambda V(x)+Z(x))|u|^{p}\right) d x
$$

for all $u \in E_{\lambda}(K)$ and $\lambda \geq 1$ or equivalently

$$
M_{0}|u|_{p, K}^{p} \leq\|u\|_{\lambda, K}^{p} \quad \text { for all } u \in E_{\lambda}(K) \text { and } \lambda \geq 1,
$$

where $M_{0}$ is a positive constant and $|u|_{p, K}^{p}=\int_{K}|u|^{p} d x$.

The next result is an immediate consequence of the above considerations.

Lemma 2.1. There exist $\delta_{0}, \nu_{0}>0$ such that for all open sets $K \subset \mathbb{R}^{N}$

$$
\delta_{0}\|u\|_{\lambda, K}^{p} \leq\|u\|_{\lambda, K}^{p}-\nu_{0}|u|_{p, K}^{p} \quad \text { for all } u \in E_{\lambda}(K) \text { and } \lambda \geq 1 \text {. }
$$

We recall the second concentration-compactness principle of Lions [34].

LEMma $2.2([34])$. Let $\left\{u_{n}\right\} \subset W^{1, p}\left(\mathbb{R}^{N}\right)$ be a bounded sequence which weakly converges to $u$ in $L^{p^{*}}\left(\mathbb{R}^{N}\right)$. If $\left\{u_{n}\right\}$ is a subsequence such that $\left.\left|u_{n}\right|\right|^{*} \rightarrow \nu$ and $\left|\nabla u_{n}\right|^{p} \rightarrow \mu$ for some measures $\nu$ and $\mu$, then there are sequences of points $\left\{x_{n}\right\} \subset \mathbb{R}^{N}$ and $\left\{\nu_{n}\right\} \subset[0, \infty)$ satisfying

(a) $\nu=|u|^{p^{*}}+\sum_{i \in I} \delta_{x_{i}} \nu_{i}, \nu_{i}>0$, 
(b) $\mu \geq|\nabla u|^{p}+\sum_{i \in I} \delta_{x_{i}} \mu_{i}, \mu_{i}>0$,

(c) $\mu_{i} \geq S \nu_{i}^{p / p^{*}}$,

where $I$ is the index set and $S$ is the best Sobolev constant, i.e.

$$
S=\inf \left\{\int_{\mathbb{R}^{N}}|\nabla u|^{p} d x: \int_{\mathbb{R}^{N}}|u|^{p^{*}} d x=1\right\},
$$

$x_{j} \in \mathbb{R}^{N}, \delta_{x_{i}}$ are Dirac measures at $x_{i}$ and $\mu_{i}, \nu_{i}$ are constants.

To finish this section, in what follows, for each $j \in\{1, \ldots, k\}$, we fix a bounded open subset $\Omega_{j}^{\prime}$ with smooth boundary such that

(a) $\overline{\Omega_{j}} \subset \Omega_{j}^{\prime}$;

(b) $\overline{\Omega_{j}^{\prime}} \cap \overline{\Omega_{j}^{\prime}}=\emptyset$ for all $j \neq \bar{j}$.

\section{Preliminaries}

In this section, we adapt for our case some arguments developed in papers [23] and [26]. Let $\nu_{0}>0$ be the constant given in Lemma 2.1, $k>\theta(\theta-p)^{-1}>1$, $a>0$ verify $\alpha f(a)+a^{p^{*}-1}=k^{-1} a^{p-1} \nu_{0}$, and $\widetilde{f}, \widetilde{F}: \mathbb{R} \rightarrow \mathbb{R}$ be the functions

$$
\widetilde{f}(s)=\left\{\begin{array}{ll}
\alpha f(s)+s^{p^{*}-1}, & \text { if } s \leq a, \\
k^{-1} \nu_{0} s^{p-1}, & \text { if } s>a,
\end{array} \quad \text { and } \quad \widetilde{F}(s)=\int_{0}^{s} \tilde{f}(\tau) d \tau .\right.
$$

From now on, we fix a non-empty subset $\Gamma \subset\{1, \ldots, k\}$ and

$$
\Omega_{\Gamma}=\bigcup_{j \in \Gamma} \Omega_{j}, \quad \Omega_{\Gamma}^{\prime}=\bigcup_{j \in \Gamma} \Omega_{j}^{\prime}, \quad \chi_{\Gamma}(x)= \begin{cases}1 & \text { for } x \in \Omega_{\Gamma}^{\prime} \\ 0 & \text { for } x \notin \Omega_{\Gamma}^{\prime}\end{cases}
$$

and functions

$$
\begin{aligned}
g(x, s) & =\chi_{\Gamma}(x)\left(\alpha f(s)+s^{p^{*}-1}\right)+\left(1-\chi_{\Gamma}(x)\right) \widetilde{f}(s) \\
G(x, s) & =\int_{0}^{s} g(x, \tau) d \tau=\chi_{\Gamma}(x) F(s)+\left(1-\chi_{\Gamma}(x)\right) \widetilde{F}(s) .
\end{aligned}
$$

Notice that, using $\left(\mathrm{f}_{1}\right)-\left(\mathrm{f}_{4}\right)$, it is easy to check that

$\left(\mathrm{g}_{1}\right) g(z, s)=\alpha f(s)+s^{p^{*}-1}=o\left(s^{p-1}\right)$, near the origin, uniformly in $z \in \mathbb{R}^{N}$.

$\left(\mathrm{g}_{2}\right) g(z, s) \leq \alpha f(s)+s^{p^{*}-1}$ for all $s>0, z \in \mathbb{R}^{N}$.

$\left(\mathrm{g}_{3}\right) \quad$ (i) $0<\theta G(x, s) \leq g(x, s) s$ for each $x \in \Omega_{\Gamma}^{\prime}, s>0$;

(ii) $0 \leq G(x, s) \leq k^{-1} p^{-1} \nu_{0} s^{p}$ and $0 \leq g(x, s) s \leq k^{-1} \nu_{0} s^{p}$ for each $x \in \mathbb{R}^{N} \backslash \Omega_{\Gamma}^{\prime}, s>0$.

$\left(\mathrm{g}_{4}\right)$ The function $g(z, s) / s^{p-1}$ is increasing for $s>0$ for each fixed $z$. 
Moreover, let $\Phi_{\lambda}: E_{\lambda} \rightarrow R$ denote the functional given by

$$
\begin{aligned}
\Phi_{\lambda}(u):=\frac{1}{p} \int_{\mathbb{R}^{N}}\left(|\nabla u|^{p}+(\lambda V(x)\right. & \left.+Z(x))|u|^{p}\right) d x \\
& +\frac{b}{2}\left(\int_{\mathbb{R}^{N}} \frac{1}{p}|\nabla u|^{p} d x\right)^{2}-\int_{\mathbb{R}^{N}} G(x, u) d x .
\end{aligned}
$$

Under conditions $\left(\mathrm{V}_{1}\right),\left(\mathrm{V}_{2}\right),\left(\mathrm{f}_{1}\right)$ and $\left(\mathrm{f}_{2}\right), \Phi_{\lambda} \in C^{1}\left(E_{\lambda}, \mathbb{R}\right)$ and its critical points are nonnegative weak solutions of

$$
-\left(1+b \int_{\mathbb{R}^{N}}|\nabla u|^{p} d x\right) \Delta_{p} u+(\lambda V(x)+Z(x))|u|^{p-2} u=g(x, u), \quad x \in \mathbb{R}^{N} .
$$

We remark that $\widetilde{f}(s)=\alpha f(s)+s^{p^{*}-1}$ for $s \in[0, a]$ and a critical point $u_{\lambda}$ of $\Phi_{\lambda}(u)$ is a solution of problem (1.1) if and only if $u_{\lambda}(x) \leq a$ in $\mathbb{R}^{N} \backslash \Omega_{\Gamma}^{\prime}$.

\section{Behavior of (PS)-sequences and the study of some energy levels}

Recall that we say that a sequence $\left(u_{n}\right)$ is a (PS)-sequence at level $c\left((\mathrm{PS})_{c^{-}}\right.$ sequence, for short) if $\Phi_{\lambda}\left(u_{n}\right) \rightarrow c$ and $\Phi_{\lambda}^{\prime}\left(u_{n}\right) \rightarrow 0 . \Phi_{\lambda}$ is said to satisfy the $(\mathrm{PS})_{c}$-condition if any $(\mathrm{PS})_{c}$-sequence contains a convergent subsequence.

Remark 4.1. By the definition of the functions $f$ and $F$, the Palais-Smale sequences may be assumed to be nonnegative.

The next lemma establishes that all $(\mathrm{PS})_{c}$-sequences are bounded.

Lemma 4.2. Assume that $\left(\mathrm{V}_{1}\right)-\left(\mathrm{V}_{3}\right)$ and $\left(\mathrm{f}_{1}\right)-\left(\mathrm{f}_{3}\right)$ are satisfied. Then there is a constant $M(c)>0$ independent of $\lambda$ such that, for any $(\mathrm{PS})_{c}$-sequence $\left(u_{n}\right)$ for $\Phi_{\lambda}(u)$,

$$
\begin{aligned}
& \Phi_{\lambda}\left(u_{n}\right) \rightarrow c, \\
& \Phi_{\lambda}^{\prime}\left(u_{n}\right) \rightarrow 0 \quad \text { strongly in } E_{\lambda}^{*},
\end{aligned}
$$

$\left\|u_{n}\right\|_{\lambda}^{p} \leq M(c)$ for all $n \in \mathbb{N}$.

Proof. It follows from (4.1)-(4.2) that

$$
\Phi_{\lambda}\left(u_{n}\right)-\frac{1}{\theta} \Phi_{\lambda}^{\prime}\left(u_{n}\right) u_{n}=c+o_{n}(1)+\varepsilon_{n}\left\|u_{n}\right\|_{\lambda},
$$

where $\varepsilon_{n} \rightarrow 0$ as $n \rightarrow \infty$. From (4.1)-(4.2), (g $\mathrm{g}_{3}$ ) (ii) and Lemma 2.1,

$$
\begin{aligned}
\Phi_{\lambda}\left(u_{n}\right) & -\frac{1}{\theta} \Phi_{\lambda}^{\prime}\left(u_{n}\right) u_{n} \\
= & \left(\frac{1}{p}-\frac{1}{\theta}\right) \int_{\mathbb{R}^{N}}\left(\left|\nabla u_{n}\right|^{p}+(\lambda V(x)+Z(x))\left|u_{n}\right|^{p}\right) d x \\
& +\frac{b}{p}\left(\frac{1}{2 p}-\frac{1}{\theta}\right)\left(\int_{\mathbb{R}^{N}}\left|\nabla u_{n}\right|^{p} d x\right)^{2}+\int_{\mathbb{R}^{N}}\left[\frac{1}{\theta} g\left(x, u_{n}\right) u_{n}-G\left(x, u_{n}\right)\right] d x \\
\geq & \left(\frac{1}{p}-\frac{1}{\theta}\right) \int_{\mathbb{R}^{N}}\left(\left|\nabla u_{n}\right|^{p}+(\lambda V(x)+Z(x))\left|u_{n}\right|^{p}\right) d x
\end{aligned}
$$




$$
\begin{aligned}
& +\int_{\mathbb{R}^{N} \backslash \Omega_{\Gamma}^{\prime}}\left[\frac{1}{\theta} g\left(x, u_{n}\right) u_{n}-G\left(x, u_{n}\right)\right] d x \\
\geq & \left(\frac{1}{p}-\frac{1}{\theta}\right) \int_{\mathbb{R}^{N}}\left(\left|\nabla u_{n}\right|^{p}+(\lambda V(x)+Z(x))\left|u_{n}\right|^{p}\right) x-\int_{\mathbb{R}^{N} \backslash \Omega_{\Gamma}^{\prime}} G\left(x, u_{n}\right) d x \\
\geq & \left(\frac{1}{p}-\frac{1}{\theta}\right) \int_{\mathbb{R}^{N}}\left(\left|\nabla u_{n}\right|^{p}+(\lambda V(x)+Z(x))\left|u_{n}\right|^{p}\right) d x-\frac{1}{k p} \nu_{0} \int_{\mathbb{R}^{N}}\left|u_{n}\right|^{p} d x \\
\geq & \left(\frac{1}{p}-\frac{1}{\theta}\right)\left(\left\|u_{n}\right\|_{\lambda}^{p}-\nu_{0}\left|u_{n}\right|_{p}^{p}\right) \geq\left(\frac{1}{p}-\frac{1}{\theta}\right) \delta_{0}\left\|u_{n}\right\|_{\lambda}^{p} .
\end{aligned}
$$

This fact implies that

$$
\left(\frac{1}{p}-\frac{1}{\theta}\right) \delta_{0}\left\|u_{n}\right\|_{\lambda}^{p} \leq c+o_{n}(1)+\varepsilon_{n}\left\|u_{n}\right\|_{\lambda} .
$$

Therefore

$$
\limsup _{n \rightarrow \infty}\left\|u_{n}\right\|_{\lambda}^{p} \leq\left(\frac{1}{p}-\frac{1}{\theta}\right)^{-1} \delta_{0}^{-1} c
$$

From which it follows that there exists $M(c)>0$ such that $\left\|u_{n}\right\|_{\lambda}^{p} \leq M(c)$ for all $n \in \mathbb{N}$.

Next, for each fixed $j \in \Gamma$, let us denote by $c_{j}$ the minimax level of the mountain pass theorem with the functional $I_{j}: W_{0}^{1, p}\left(\Omega_{j}\right) \rightarrow \mathbb{R}$, given by

$$
\begin{aligned}
I_{j}(u)=\frac{1}{p} \int_{\Omega_{j}}\left(|\nabla u|^{p}+Z(x)|u|^{p}\right) d x+ & \frac{b}{2}\left(\int_{\Omega_{j}} \frac{1}{p}|\nabla u|^{p} d x\right)^{2} \\
& -\alpha \int_{\Omega_{j}} F\left(u_{+}\right) d x-\frac{1}{p^{*}} \int_{\Omega_{j}}\left(u_{+}\right)^{p^{*}} d x .
\end{aligned}
$$

It is well-known that the critical points of $I_{j}$ are weak solutions of the following problem:

$$
\begin{cases}-\left(1+b \int_{\Omega_{j}}|\nabla u|^{p} d x\right) \Delta u+Z(x) u^{p-1}= & \alpha f(u)+u^{p^{*}-1}, \\ u>0, & x \in \Omega_{j}, j \in \Gamma, \\ \left.u\right|_{\partial \Omega_{j}}=0 . & x \in \Omega_{j},\end{cases}
$$

The technique that we shall apply in order to prove Theorem 1.1 includes the comparison between some energy levels of the functional associated with (1.1) with the energy levels associated with other auxiliary problems related to (1.1), as well as the study of the behaviour of some $(\mathrm{PS})_{c}$-sequences. In this regard we prove the following results.

Lemma 4.3. There exists $\alpha^{*}>0$ such that, for all $\alpha \geq \alpha^{*}$, we have

$$
c_{j} \in\left(0, \frac{1}{2 p(k+1)} S^{N / p}\right) \quad \text { for all } j \in\{1, \ldots, k\} .
$$


Proof. We fix a nonnegative function $\varphi_{j} \in W_{0}^{1, p}\left(\Omega_{j}\right) \backslash\{0\}$ for each $j \in$ $\{1, \ldots, k\}$. Observe that there exists $t_{\alpha, j} \in(0,+\infty)$ such that

$$
c_{j} \leq I_{j}\left(t_{\alpha, j} \varphi_{j}\right)=\max _{t \geq 0} I_{j}\left(t \varphi_{j}\right)
$$

and thus, by $\left(f_{5}\right)$, we have

$$
\begin{aligned}
& t_{\alpha, j}^{p} \int_{\Omega_{j}}\left(\left|\nabla \varphi_{j}\right|^{p}+Z(x)\left|\varphi_{j}\right|^{p}\right) d x+t_{\alpha, j}^{2 p} b\left(\int_{\Omega_{j}}\left|\nabla \varphi_{j}\right|^{p} d x\right)^{2} \\
& \quad=\alpha \int_{\Omega_{j}} f\left(t_{\alpha, j} \varphi_{j}\right) t_{\alpha, j} \varphi_{j} d x+t_{\alpha, j}^{p^{*}} \int_{\Omega_{j}}\left|\varphi_{j}\right|^{p^{*}} d x \\
& \quad \geq \alpha \int_{\Omega_{j}} f\left(t_{\alpha, j} \varphi_{j}\right) t_{\alpha, j} \varphi_{j} d x \geq \alpha \varsigma t_{\alpha, j}^{\iota} \int_{\Omega_{j}}\left|\varphi_{j}\right|^{\iota} d x .
\end{aligned}
$$

If $\left|t_{\beta, j}\right| \leq 1$, by (4.4), we have

$$
t_{\alpha, j}^{p} \int_{\Omega_{j}}\left(\left|\nabla \varphi_{j}\right|^{p}+Z(x)\left|\varphi_{j}\right|^{p}\right) d x+t_{\alpha, j}^{p} b\left(\int_{\Omega_{j}}\left|\nabla \varphi_{j}\right|^{p} d x\right)^{2} \geq \alpha \varsigma t_{\alpha, j}^{\iota} \int_{\Omega_{j}}\left|\varphi_{j}\right|^{\iota} d x .
$$

This fact implies that

$$
t_{\alpha, j} \leq\left[\frac{\int_{\Omega_{j}}\left(\left|\nabla \varphi_{j}\right|^{2}+Z(x)\left|\varphi_{j}\right|^{2}\right) d x+b\left(\int_{\Omega_{j}}\left|\nabla \varphi_{j}\right|^{p} d x\right)^{2}}{\alpha \varsigma \int_{\Omega_{j}}\left|\varphi_{j}\right|^{\iota} d x}\right]^{1 /(\iota-p)} .
$$

If $\left|t_{\beta, j}\right| \geq 1$, by (4.4), one has

$$
t_{\alpha, j}^{2 p} \int_{\Omega_{j}}\left(\left|\nabla \varphi_{j}\right|^{p}+Z(x)\left|\varphi_{j}\right|^{p}\right) d x+t_{\alpha, j}^{2 p} b\left(\int_{\Omega_{j}}\left|\nabla \varphi_{j}\right|^{p} d x\right)^{2} \geq \alpha \varsigma t_{\alpha, j}^{\iota} \int_{\Omega_{j}}\left|\varphi_{j}\right|^{\iota} d x .
$$

This fact implies that

$$
t_{\alpha, j} \leq\left[\frac{\int_{\Omega_{j}}\left(\left|\nabla \varphi_{j}\right|^{2}+Z(x)\left|\varphi_{j}\right|^{2}\right) d x+b\left(\int_{\Omega_{j}}\left|\nabla \varphi_{j}\right|^{p} d x\right)^{2}}{\alpha \varsigma \int_{\Omega_{j}}\left|\varphi_{j}\right|^{\iota} d x}\right]^{1 /(\iota-2 p)} .
$$

Using the above limit, we have $t_{\alpha, j} \rightarrow 0$ as $\alpha \rightarrow+\infty$. This fact implies that $I_{j}\left(t_{\alpha, j} \varphi_{j}\right) \rightarrow 0$ as $\alpha \rightarrow+\infty$, whence it follows that there exists $\alpha^{*}>0$ such that

$$
c_{j} \in\left(0, \frac{1}{2 p(k+1)} S^{N / p}\right) \quad \text { for all } j \in\{1, \ldots, k\} \text { and all } \alpha \in\left[\alpha^{*},+\infty\right) .
$$

REMARK 4.4. In particular, Lemma 4.3 implies that

$$
\sum_{j=1}^{k} c_{j} \in\left(0, S^{N / p} /(2 p)\right)
$$

The above result is very important, as we show in the following lemma. 
Lemma 4.5. Assume that $\left(\mathrm{V}_{1}\right)-\left(\mathrm{V}_{3}\right)$ and $\left(\mathrm{f}_{1}\right)-\left(\mathrm{f}_{5}\right)$ are satisfied. For any $\lambda \geq 1, \Phi_{\lambda}$ satisfies the $(\mathrm{PS})_{c}$-condition, for all $c \in\left(0,1 / 2 p S^{N / p}\right)$, that is any (PS $)_{c}$-sequence $\left(u_{n}\right) \subset E_{\lambda}$ has a strongly convergent subsequence in $E_{\lambda}$.

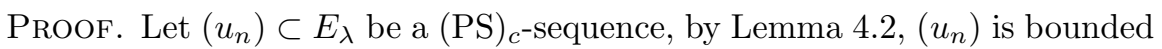
in $E_{\lambda}$ and we may assume the following facts:

$$
\begin{aligned}
& u_{n} \rightarrow u \text { weakly in } E_{\lambda} \text { and } W^{1, p}\left(\mathbb{R}^{N}\right), \\
& u_{n} \rightarrow u \text { strongly in } L_{\text {loc }}^{p}\left(\mathbb{R}^{N}\right) \text { for all } p \in\left[1, p^{*}\right), \\
&\left|u_{n}\right|^{p^{*}} \rightarrow \nu=|u|^{p^{*}}+\sum_{i \in I} \delta_{x_{i}} \nu_{i}, \quad \nu_{i}>0, \\
&\left|\nabla u_{n}\right|^{p} \rightarrow \mu \geq|\nabla u|^{p}+\sum_{i \in I} \delta_{x_{j}} \mu_{i}, \quad \mu_{i}>0 .
\end{aligned}
$$

First, we claim that

$$
\int_{\mathbb{R}^{N}} g\left(x, u_{n}\right) u_{n} d x \rightarrow \int_{\mathbb{R}^{N}} g(x, u) u d x \quad \text { as } n \rightarrow \infty .
$$

In fact, let $x_{i}$ be a singular point of the measures $\mu$ and $\nu$. We define a function $\phi \in C_{0}^{\infty}\left(\mathbb{R}^{N}\right)$ such that $\phi(x)=1$ in $B\left(x_{i}, \varepsilon\right), \phi(x)=0$ in $\mathbb{R}^{N} \backslash B\left(x_{i}, 2 \varepsilon\right)$ and $|\nabla \phi| \leq 2 / \varepsilon$ in $\mathbb{R}^{N}$. Set $\psi_{n}=u_{n} \phi$, then $\left\{\psi_{n}\right\}$ is bounded in $E_{\lambda}$. Obviously, $\left\langle\Phi_{\lambda}^{\prime}\left(u_{n}\right), \psi_{n}\right\rangle \rightarrow 0$, i.e.

$$
\begin{aligned}
& -\lim _{n \rightarrow \infty}\left[\int_{\mathbb{R}^{N}} u_{n}\left|\nabla u_{n}\right|^{p-2} \nabla u_{n} \nabla \phi d x\right. \\
& \left.+b\left(\int_{\mathbb{R}^{N}} \frac{1}{p}\left|\nabla u_{n}\right|^{p} d x\right)\left(\int_{\mathbb{R}^{N}} u_{n}\left|\nabla u_{n}\right|^{p-2} \nabla u_{n} \nabla \phi d x\right)\right] \\
= & \lim _{n \rightarrow \infty}\left\{\int_{\mathbb{R}^{N}}\left|\nabla u_{n}\right|^{p} \phi d x+\int_{\mathbb{R}^{N}}(\lambda V(x)+Z(x))\left|u_{n}\right|^{p} \phi d x\right. \\
& \left.+b\left(\int_{\mathbb{R}^{N}} \frac{1}{p}\left|\nabla u_{n}\right|^{p} d x\right)\left(\int_{\mathbb{R}^{N}}\left|\nabla u_{n}\right|^{p} \phi d x\right)-\int_{\mathbb{R}^{N}} g\left(x, u_{n}\right) u_{n} \phi d x\right\} .
\end{aligned}
$$

By definition of $\phi$ and due to strong convergence $u_{n} \rightarrow u$ in $L_{\text {loc }}^{p}\left(\mathbb{R}^{N}\right)$ for $p \in$ $\left[1, p^{*}\right)$, one can obtain

$$
\int_{\mathbb{R}^{N}} f\left(u_{n}\right) u_{n} \phi d x \rightarrow 0 \quad \text { as } n \rightarrow \infty
$$

From Lemma 4.2 and the Hölder inequality, we have that

$$
\begin{aligned}
0 & \leq\left.\lim _{\varepsilon \rightarrow 0} \lim _{n \rightarrow \infty}\left|\int_{\mathbb{R}^{N}} u_{n}\right| \nabla u_{n}\right|^{p-2} \nabla u_{n} \nabla \phi d x \mid \\
& \leq\left. C \lim _{\varepsilon \rightarrow 0} \lim _{n \rightarrow \infty} \int_{\mathbb{R}^{N}}\left|u_{n}\right| \nabla u_{n}\right|^{p-1} \nabla \phi \mid d x
\end{aligned}
$$




$$
\begin{aligned}
& \leq C \lim _{\varepsilon \rightarrow 0} \lim _{n \rightarrow \infty}\left[\left(\int_{\mathbb{R}^{N}}\left|\nabla u_{n}\right|^{p} d x\right)^{(p-1) / p}\left(\int_{\mathbb{R}^{N}}\left|u_{n} \nabla \phi\right|^{p} d x\right)^{1 / p}\right] \\
& \leq C \lim _{\varepsilon \rightarrow 0}\left(\int_{B\left(x_{i}, 2 \varepsilon\right)}|u|^{p^{*}} d x\right)^{1 / p^{*}}=0
\end{aligned}
$$

and

$$
\lim _{\varepsilon \rightarrow 0} \lim _{n \rightarrow \infty}\left[b\left(\int_{\mathbb{R}^{N}} \frac{1}{p}\left|\nabla u_{n}\right|^{p} d x\right)\left(\int_{\mathbb{R}^{N}} u_{n}\left|\nabla u_{n}\right|^{p-2} \nabla u_{n} \nabla \phi d x\right)\right]=0 .
$$

From inequalities (4.7)-(4.9) and condition $\left(\mathrm{g}_{2}\right)$, we get

$$
\begin{aligned}
0 \geq & \lim _{\varepsilon \rightarrow 0} \lim _{n \rightarrow \infty}\left\{\int_{\mathbb{R}^{N}}\left|\nabla u_{n}\right|^{p} \phi d x+\int_{\mathbb{R}^{N}}(\lambda V(x)+Z(x))\left|u_{n}\right|^{p} \phi d x\right. \\
& +b\left(\int_{\mathbb{R}^{N}} \frac{1}{p}\left|\nabla u_{n}\right|^{p} d x\right)\left(\int_{\mathbb{R}^{N}}\left|\nabla u_{n}\right|^{p} \phi d x\right) \\
& \left.-\alpha \int_{\mathbb{R}^{N}} f\left(u_{n}\right) u_{n} \phi d x-\int_{\mathbb{R}^{N}} u_{n}^{p^{*}} \phi d x\right\} \\
\geq & \lim _{\varepsilon \rightarrow 0} \lim _{n \rightarrow \infty}\left[\int_{\mathbb{R}^{N}}\left|\nabla u_{n}\right|^{p} \phi d x-\int_{\mathbb{R}^{N}} u_{n}^{p^{*}} \phi d x\right]=\mu_{i}-\nu_{i} .
\end{aligned}
$$

Combining this with Lemma 2.2, we obtain $\nu_{i} \geq S \nu_{i}^{p / p^{*}}$. This result implies that

(I) $\nu_{i}=0$ or

(II) $\nu_{i} \geq S^{N / p}$.

If the second case $\nu_{i} \geq S^{N / p}$ holds, for some $i \in I$, then by using Lemma 2.2 and the Hölder inequality, we have

$$
\begin{aligned}
c & =\lim _{n \rightarrow \infty}\left(\Phi_{\lambda}\left(u_{n}\right)-\frac{1}{2 p}\left\langle\Phi_{\lambda}^{\prime}\left(u_{n}\right), u_{n}\right\rangle\right) \\
& =\frac{1}{2 p} \int_{\mathbb{R}^{N}}\left(\left|\nabla u_{n}\right|^{p}+(\lambda V(x)+Z(x))\left|u_{n}\right|^{p}\right) d x+\int_{\mathbb{R}^{N}}\left(\frac{1}{2 p} g\left(x, u_{n}\right)-G\left(x, u_{n}\right)\right) d x \\
& \geq \frac{1}{2 p} \int_{\mathbb{R}^{N}}\left|\nabla u_{n}\right|^{p} d x=\frac{1}{2 p} \int_{\mathbb{R}^{N}} d \mu \\
& \geq \frac{1}{2 p} \int_{\mathbb{R}^{N}}|\nabla u|^{p} d x+\frac{1}{2 p} \mu_{i} \geq \frac{1}{2 p} S \nu_{i}^{p / p^{*}} \geq \frac{1}{2 p} S^{N / p} .
\end{aligned}
$$

This is impossible. Consequently, $\nu_{i}=0$ for all $i \in I$ and hence

$$
u_{n} \rightarrow u \quad \text { in } L_{\mathrm{loc}}^{p^{*}}\left(\mathbb{R}^{N}\right) .
$$

In order to prove (4.6), we will prove that the possible concentration of mass at infinity. By hypothesis, for any bounded sequence $\left(\varphi_{n}\right) \subset E_{\lambda}$, we have $\Phi_{\lambda}^{\prime}\left(u_{n}\right) \varphi_{n}=o_{n}(1)$. Let us choose a special $\varphi_{n}$ for our purposes:

$$
\varphi_{n}(x)=\eta(x) u_{n}(x)
$$


where $\eta \in C^{\infty}\left(\mathbb{R}^{N}\right)$ is given by

$$
\begin{gathered}
\eta(x)= \begin{cases}1 & \text { for } x \in B_{R}^{c}(0), \\
0 & \text { for } x \in B_{R / 2}(0),\end{cases} \\
\eta(x) \in[0,1] \quad \text { with } \Omega_{\Gamma}^{\prime} \subset B_{R / 2}(0) .
\end{gathered}
$$

Here and below $B_{R}^{c}(0)=\left\{x \in \mathbb{R}^{N}:|x| \geq R\right\}$. Using the above data and adapting arguments used in [23, Lemma 1.1] one proves that, for each $\varepsilon>0$, there exists $R>0$ such that

$$
\int_{\left\{x \in \mathbb{R}^{N}:|x| \geq R\right\}}\left(\left|\nabla u_{n}\right|^{p}+(\lambda V(x)+Z(x))\left|u_{n}\right|^{p}\right) d x<\varepsilon
$$

and

$$
\left(\int_{\left\{x \in \mathbb{R}^{N}:|x| \geq R\right\}}\left|\nabla u_{n}\right|^{p} d x\right)^{2}<\varepsilon,
$$

for large enough $n \in \mathbb{N}$. From (4.11)-(4.13), we obtain (4.6) holds. Thus, from the Brezis-Lieb lemma [13] and the weak lower semicontinuity of the norm we have

$$
\begin{aligned}
o(1)\left\|v_{n}\right\|= & \left\langle\Phi_{\lambda}^{\prime}\left(u_{n}\right), u_{n}\right\rangle=\int_{\mathbb{R}^{N}}\left(\left|\nabla u_{n}\right|^{p}+(\lambda V(x)+Z(x))\left|u_{n}\right|^{p}\right) d x \\
& +\frac{b}{p}\left(\int_{\mathbb{R}^{N}}\left|\nabla u_{n}\right|^{p} d x\right)^{2}-\int_{\mathbb{R}^{N}} g\left(x, u_{n}\right) d x \\
\geq & \left\|u_{n}-u\right\|_{\lambda}^{p}+\|u\|_{\lambda}^{p}+\frac{b}{p}\left(\int_{\mathbb{R}^{N}}|\nabla u|^{p} d x\right)^{2}-\int_{\mathbb{R}^{N}} g(x, u) d x \\
= & \left\|u_{n}-u\right\|_{\lambda}^{p}+o(1)\|u\|,
\end{aligned}
$$

since $\Phi_{\lambda}^{\prime}(u)=0$. Thus we proved that $\left\{u_{n}\right\}$ strongly converges to $u$ in $E_{\lambda}$. This completes the proof of Lemma 4.5.

REMARK 4.6. Since $\Phi_{\lambda}$ verifies the mountain pass geometry, the above results imply the existence of a nontrivial critical point to $\Phi_{\lambda}$.

Our next step is to study the behavior of a $(\mathrm{PS})_{\infty, c^{-}}$-sequence, that is, a sequence $\left(u_{n}\right) \subset W^{1, p}\left(\mathbb{R}^{N}\right)$ that satisfies

$$
u_{n} \subset E_{\lambda} \quad \text { and } \quad \lambda_{n} \rightarrow \infty, \quad \Phi_{\lambda_{n}}\left(u_{n}\right) \rightarrow c, \quad\left\|\Phi_{\lambda_{n}}^{\prime}\left(u_{n}\right)\right\|_{\lambda_{n}}^{*} \rightarrow 0 .
$$

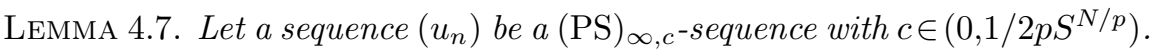
Then, for some subsequence, still denoted by $\left(u_{n}\right)$, there exists $u \in W^{1, p}\left(\mathbb{R}^{N}\right)$ such that

$$
u_{n} \rightarrow u \quad \text { weakly in } E_{\lambda} \text { and } W^{1, p}\left(\mathbb{R}^{N}\right) \quad \text { as } n \rightarrow \infty .
$$

Moreover, 
(a) $u \equiv 0$ in $\mathbb{R}^{N} \backslash \Omega_{\Gamma}$ and $u$ is a nonnegative solution of

$$
\left\{\begin{array}{lr}
-\left(1+b \int_{\mathbb{R}^{N}}|\nabla u|^{p} d x\right) \Delta_{p} u+Z(x)|u|^{p-2} u=\alpha f(u)+|u|^{p^{*}-2} u \\
& \text { in } \in \Omega_{j}, \\
u=0 & \text { on } \partial \Omega_{j},
\end{array}\right.
$$

for each $j \in \Gamma$;

(b) $\left\|u_{n}-u\right\|_{\lambda_{n}} \rightarrow 0$

(c) $u_{n}$ also satisfies

$$
\begin{aligned}
& \lambda_{n} \int_{\mathbb{R}^{N}} V(x)\left|u_{n}\right|^{p} d x \rightarrow 0, \quad\left\|u_{n}-u\right\|_{\lambda_{n}, \mathbb{R}^{N} \backslash \Omega_{\Gamma}}^{p} \rightarrow 0, \\
& \left\|u_{n}\right\|_{\lambda_{n}, \Omega_{j}^{\prime}}^{p} \rightarrow \int_{\Omega_{j}}\left(|\nabla u|^{p}+Z(x)|u|^{p}\right) d x \quad \text { for all } j \in \Gamma .
\end{aligned}
$$

Proof. Similarly to the proof of Lemma 4.2, we can prove that there exists $M(c)>0$ such that

$$
\left\|u_{n}\right\|_{\lambda}^{p} \leq M(c) \text { for all } n \in \mathbb{N} .
$$

Thus $\left(u_{n}\right)$ is a bounded sequence in $W^{1, p}\left(\mathbb{R}^{N}\right)$ and, for some subsequence, still denoted by $\left(u_{n}\right)$, we may assume that for some $u \in W^{1, p}\left(\mathbb{R}^{N}\right)$

$$
\begin{array}{rlrl}
u_{n} & \rightarrow u & \text { weakly in } E_{\lambda} \text { and } W^{1, p}\left(\mathbb{R}^{N}\right), \\
u_{n} & \rightarrow u & \text { strongly in } L_{\text {loc }}^{p}\left(\mathbb{R}^{N}\right) \text { for all } p \in\left[1, p^{*}\right), \\
u_{n}(x) & \rightarrow u(x) & & \text { a.e. in } \mathbb{R}^{N} .
\end{array}
$$

Using once more similar arguments explored in Lemma 4.5, we get

$$
u_{n} \rightarrow u \quad \text { in } E_{\lambda} .
$$

To show (a), we fix the set $C_{m}=\left\{x \in \mathbb{R}^{N}: V(x) \geq 1 / m\right\}$. Then

$$
\begin{aligned}
\int_{C_{m}}\left|u_{n}\right|^{p} d x & \leq \frac{m}{\lambda_{n}} \int_{\mathbb{R}^{N}} \lambda_{n} V(x)\left|u_{n}\right|^{p} d x \\
& \leq \frac{m}{\lambda_{n}} \int_{\mathbb{R}^{N}}\left(\left|\nabla u_{n}\right|^{p}+\left(\lambda_{n} V(x)+Z(x)\right)\left|u_{n}\right|^{p}\right) d x=\frac{m}{\lambda_{n}}\left\|u_{n}\right\|_{\lambda_{n}}^{p} .
\end{aligned}
$$

The last inequality together with Fatou's lemma imply

$$
\int_{C_{m}}|u|^{p} d x=0 \quad \text { for all } m \in \mathbb{N} .
$$

Thus $u(x)=0$ on $\bigcup_{m=1}^{+\infty} C_{m}=\mathbb{R}^{N} \backslash \bar{\Omega}$ and we can assert that $\left.u\right|_{\Omega_{j}} \in W_{0}^{1, p}\left(\Omega_{j}\right)$ for all $j \in\{1, \ldots, k\}$. 
Once we have proved that $\Phi_{\lambda_{n}}^{\prime}\left(u_{n}\right) \varphi \rightarrow 0$ for $\varphi \in C_{0}^{\infty}\left(\Omega_{j}\right)(j \in\{1, \ldots, k\})$, it follows from (4.15) that

$$
\begin{aligned}
0= & \int_{\Omega_{j}}\left(|\nabla u|^{p-2} \nabla u \nabla \varphi+Z(x)|u|^{p-2} u \varphi\right) d x \\
& +b\left(\int_{\mathbb{R}^{N}} \frac{1}{p}|\nabla u|^{p} d x\right) \int_{\Omega_{j}}|\nabla u|^{p-2} \nabla u \nabla \varphi d x-\int_{\Omega_{j}} g(x, u) \varphi d x,
\end{aligned}
$$

showing that $u$ is a solution to (4.14) for each $j \in \Gamma$.

For $j \in\{1, \ldots, k\} \backslash \Gamma$, setting $\varphi(x)=u(x)$, we have

$$
\int_{\Omega_{j}}\left(|\nabla u|^{p}+Z(x)|u|^{p}\right) d x+\frac{b}{p}\left(\int_{\Omega_{j}}|\nabla u|^{p} d x\right)^{2}-\int_{\Omega_{j}} \widetilde{f}(u) u d x=0 .
$$

From Lemma 2.1 and the fact that $\widetilde{f}(s) s \leq \nu_{0} s^{p}$ for all $s \in \mathbb{R}$, we have

$$
\begin{aligned}
& \delta_{0}\|u\|_{\lambda, \Omega_{j}}^{p} \leq\|u\|_{\lambda, \Omega_{j}}^{p}-\nu_{0}|u|_{p, \Omega_{j}^{\prime}}^{p} \\
& \quad \leq \int_{\Omega_{j}}\left(|\nabla u|^{p}+Z(x)|u|^{p}\right) d x+\frac{b}{p}\left(\int_{\Omega_{j}}|\nabla u|^{p} d x\right)^{2}-\int_{\Omega_{j}} \widetilde{f}(u) u d x=0 .
\end{aligned}
$$

Thus, $u=0$ in $\Omega_{j}$ for $j \in\{1, \ldots, k\} \backslash \Gamma$ showing that (a) holds.

For (b), we use [46, Lemma 4.2] to get the following inequality:

$$
\begin{aligned}
\| u_{n} & -u \|_{\lambda_{n}}^{p}+b\left(\int_{\mathbb{R}^{N}} \frac{1}{p}\left|\nabla u_{n}\right|^{p} d x\right) \int_{\mathbb{R}^{N}}\left|\nabla u_{n}\right|^{p-2} \nabla u_{n}\left(\nabla u_{n}-\nabla u\right) d x \\
& -b\left(\int_{\mathbb{R}^{N}} \frac{1}{p}|\nabla u|^{p} d x\right) \int_{\mathbb{R}^{N}}|\nabla u|^{p-2} \nabla u\left(\nabla u-\nabla u_{n}\right) d x \\
& -\int_{\mathbb{R}^{N} \backslash \Omega_{\Gamma}^{\prime}}\left(\widetilde{f}\left(u_{n}\right)-\widetilde{f}(u)\right)\left(u_{n}-u\right) d x-\int_{\Omega_{\Gamma}^{\prime}}\left(f\left(u_{n}\right)-f(u)\right)\left(u_{n}-u\right) d x \\
\leq & \Phi_{\lambda_{n}}^{\prime}\left(u_{n}\right)\left(u_{n}-u\right)-\Phi_{\lambda_{n}}^{\prime}(u)\left(u_{n}-u\right) .
\end{aligned}
$$

Since $u_{n} \rightarrow u$ in $L^{p}\left(\Omega_{J}^{\prime}\right)$ and due to assumptions $\left(\mathrm{f}_{1}\right)-\left(\mathrm{f}_{2}\right)$, we have

$$
\int_{\Omega_{\Gamma}^{\prime}}\left(f\left(u_{n}\right)-f(u)\right)\left(u_{n}-u\right) d x=o_{n}(1) .
$$

On the other hand,

$$
\left|\Phi_{\lambda_{n}}^{\prime}\left(u_{n}\right)\left(u_{n}-u\right)\right| \leq\left\|\Phi_{\lambda_{n}}^{\prime}\left(u_{n}\right)\right\|_{\lambda_{n}}^{*}\left(\left\|u_{n}\right\|_{\lambda_{n}}+\|u\|_{\lambda_{n}}\right)=o_{n}(1) .
$$

By inequalities (4.16) and (4.17), we have

$$
\left\|u_{n}-u\right\|_{\lambda_{n}}^{p}+\int_{\mathbb{R}^{N} \backslash \Omega_{\Gamma}^{\prime}}\left(\widetilde{f}\left(u_{n}\right)-\widetilde{f}(u)\right)\left(u_{n}-u\right) d x \rightarrow 0 .
$$


By a similar argument as in the proof of Lemma 4.3, we obtain

$$
\begin{aligned}
\delta_{0}\left\|u_{n}-u\right\|_{\lambda_{n}}^{p} \leq & \left\|u_{n}-u\right\|_{\lambda_{n}}^{p}-\nu_{0}\left|u_{n}-u\right|_{p}^{p} \\
\leq & \int_{\mathbb{R}^{N}}\left(\left|\nabla u_{n}-\nabla u\right|^{p}+\lambda V(x)\left|u_{n}-u\right|^{p}\right) d x \\
& +\int_{\mathbb{R}^{N} \backslash \Omega_{\Gamma}^{\prime}}\left(\widetilde{f}\left(u_{n}\right)-\widetilde{f}(u)\right)\left(u_{n}-u\right) d x \leq 0 .
\end{aligned}
$$

Thus (b) is obtained.

Now we show (c). Indeed,

$$
\begin{aligned}
\int_{\mathbb{R}^{N}} \lambda_{n} V(x)\left|u_{n}\right|^{p} d x=\int_{\mathbb{R}^{N} \backslash \Omega_{\Gamma}} \lambda_{n} V(x)\left|u_{n}\right|^{p} d x \\
=\int_{\mathbb{R}^{N} \backslash \Omega_{\Gamma}} \lambda_{n} V(x)\left|u_{n}-u\right|^{p} d x \leq\left\|u_{n}-u\right\|_{\lambda_{n}}^{p} \rightarrow 0 \quad \text { as } n \rightarrow \infty .
\end{aligned}
$$

This completes the proof of Lemma 4.7.

The following lemma plays a fundamental role in the study of solutions to problem (1.1). We sketch its proof by using some arguments explored in [5] and [35], which are initiated in the Moser iterative method [39].

LEMMA 4.8. Let $u_{\lambda}$ be a family of positive solutions of (3.3) satisfying

$$
\sup _{\lambda \geq 1}\left\{\Phi_{\lambda}\left(u_{\lambda}\right)\right\}<\frac{1}{2 p} S^{N / p} .
$$

Then, there exists $\Lambda_{0}>0$ such that $\left|u_{\lambda}\right|_{L^{\infty}\left(\mathbb{R}^{N}\right) \backslash \Omega_{\Gamma}^{\prime}} \leq$ a for $\lambda \geq \Lambda_{0}$. In particular, $u_{\lambda}$ solves the original problem (1.1).

Proof. In this proof we adapt some arguments developed in Li [35, Theorem 1.11] (see also [1] and [31]). Let $\left(\lambda_{n}\right)$ be a sequence with $\lambda_{n} \rightarrow \infty$ and define $u_{n}(x)=u_{\lambda_{n}}(x)$. For any $R>0,0<r \leq R / 2$, let $\eta \in C^{\infty}\left(\mathbb{R}^{N}\right), 0 \leq \eta \leq 1$ with $\eta(x)=1$ if $|x| \geq R$ and $\eta(x)=0$ if $|x| \leq R-r$ and $|\nabla \eta| \leq 2 / r$. For each $n \in \mathbb{N}$ and $\rho>0$, put

$$
u_{l, n}(x):= \begin{cases}u_{n}(x) & \text { if } u_{n}(x) \leq \rho, \\ \rho & \text { if } u_{n}(x) \geq \rho,\end{cases}
$$

and $z_{\rho, n}:=\eta^{p} u_{\rho, n}^{p(\sigma-1)} u_{n}, w_{\rho, n}:=\eta u_{n} u_{\rho, n}^{\sigma-1}$ with $\sigma>1$ to be determined later. Taking $z_{\rho, n}$ as the test function, we have

$$
\begin{aligned}
0= & \int_{\mathbb{R}^{N}}\left(\left|\nabla u_{n}\right|^{p-2} \nabla u_{n} \nabla z_{\rho, n}+(\lambda V(x)+Z(x))\left|u_{n}\right|^{p-2} u_{n} z_{\rho, n}\right) d x \\
& +b\left(\int_{\mathbb{R}^{N}} \frac{1}{p}\left|\nabla u_{n}\right|^{p} d x\right) \int_{\mathbb{R}^{N}}\left|\nabla u_{n}\right|^{p-2} \nabla u_{n} \nabla z_{\rho, n} d x-\int_{\mathbb{R}^{N}} g\left(x, u_{n}\right) z_{\rho, n} d x,
\end{aligned}
$$


i.e.

$$
\begin{aligned}
0= & \left(1+b \int_{\mathbb{R}^{N}} \frac{1}{p}\left|\nabla u_{n}\right|^{p} d x\right) \\
& \cdot \int_{\mathbb{R}^{N}}\left(\left|\nabla u_{n}\right|^{p} \eta^{p} u_{\rho, n}^{p(\sigma-1)}+p\left|\nabla u_{n}\right|^{p-2} \nabla u_{n} \nabla \eta \eta^{p-1} u_{n} u_{\rho, n}^{p(\sigma-1)}\right. \\
& \left.+p(\sigma-1)\left|\nabla u_{n}\right|^{p-2} \nabla u_{n} \nabla u_{\rho, n} u_{n} u_{\rho, n}^{p(\sigma-1)-1} \eta^{p}\right) d x \\
& +\int_{\mathbb{R}^{N}}(\lambda V(x)+Z(x))\left|u_{n}\right|^{p} \eta^{p} u_{\rho, n}^{p(\sigma-1)} d x-\int_{\mathbb{R}^{N}} g\left(x, u_{n}\right) \eta^{p} u_{n} u_{\rho, n}^{p(\sigma-1)} d x .
\end{aligned}
$$

For simplicity, we denote

$$
A_{n}:=1+b \int_{\mathbb{R}^{N}} \frac{1}{p}\left|\nabla u_{n}\right|^{p} d x .
$$

Since $u_{n} \rightarrow u$ in $W^{1, p}\left(\mathbb{R}^{N}\right)$ with $u \neq 0$, we see that $a \leq A_{n} \leq a^{*}$ for some constant $a^{*}>0$. Therefore, we can rewrite the above equality as

$$
\begin{aligned}
A_{n} \int_{\mathbb{R}^{N}} \eta^{p} u_{\rho, n}^{p(\sigma-1)}\left|\nabla u_{n}\right|^{p} d x=\int_{\mathbb{R}^{N}} g\left(x, u_{n}\right) \eta^{p} u_{n} u_{\rho, n}^{p(\sigma-1)} d x \\
\quad-p A_{n} \int_{\mathbb{R}^{N}} \eta^{p-1} u_{n} u_{\rho, n}^{p(\sigma-1)}\left|\nabla u_{n}\right|^{p-2} \nabla u_{n} \nabla \eta d x \\
\quad-p A_{n}(\sigma-1) \int_{\mathbb{R}^{N}} u_{n} u_{\rho, n}^{p(\sigma-1)-1} \eta^{p}\left|\nabla u_{n}\right|^{p-2} \nabla u_{n} \nabla u_{\rho, n} d x \\
\quad-\int_{\mathbb{R}^{N}}(\lambda V(x)+Z(x))\left|u_{n}\right|^{p} \eta^{p} u_{\rho, n}^{p(\sigma-1)} d x .
\end{aligned}
$$

By conditions $\left(\mathrm{f}_{1}\right)-\left(\mathrm{f}_{2}\right)$, we have that for any $\tau>0$, there exists $D_{\tau}>0$ such that $g(x, s) \leq \tau s+D_{\tau} s^{p^{*}-1}$ for all $(x, s) \in \mathbb{R}^{N} \times[0,+\infty)$. Since $\tau$ can be sufficiently small, we have the following inequality:

$$
\begin{aligned}
A_{n} \int_{\mathbb{R}^{N}} \eta^{p} u_{\rho, n}^{p(\sigma-1)}\left|\nabla u_{n}\right|^{p} d x \leq & D_{\tau} \int_{\mathbb{R}^{N}} u_{n}^{p^{*}} \eta^{p} u_{\rho, n}^{p(\sigma-1)} d x \\
& -p A_{n} \int_{\mathbb{R}^{N}} \eta^{p-1} u_{n} u_{\rho, n}^{p(\sigma-1)}\left|\nabla u_{n}\right|^{p-2} \nabla u_{n} \nabla \eta d x .
\end{aligned}
$$

For each $\delta>0$, using Young's inequality, we get

$$
\begin{aligned}
A_{n} \int_{\mathbb{R}^{N}} & \eta^{p} u_{\rho, n}^{p(\sigma-1)}\left|\nabla u_{n}\right|^{p} d x=D_{\tau} \int_{\mathbb{R}^{N}} u_{n}^{p^{*}} \eta^{p} u_{\rho, n}^{p(\sigma-1)} d x \\
& +p A_{n} \delta \int_{\mathbb{R}^{N}}\left|\nabla u_{n}\right|^{p} u_{\rho, n}^{p(\sigma-1)} \eta^{p} d x+p A_{n} C_{\delta} \int_{\mathbb{R}^{N}}\left|u_{n}\right|^{p}|\nabla \eta|^{p} u_{\rho, n}^{p(\sigma-1)} d x .
\end{aligned}
$$

Choosing $\delta \in(0,1 / p)$, we have

$$
\begin{aligned}
\int_{\mathbb{R}^{N}} \eta^{p} u_{\rho, n}^{p(\sigma-1)}\left|\nabla u_{n}\right|^{p} d x \leq C \int_{\mathbb{R}^{N}} u_{n}^{p^{*}} \eta^{p} u_{\rho, n}^{p(\sigma-1)} d x & \\
& +C \int_{\mathbb{R}^{N}}\left|u_{n}\right|^{p}|\nabla \eta|^{p} u_{\rho, n}^{p(\sigma-1)} d x .
\end{aligned}
$$


On the other hand, by the Sobolev and Hölder inequalities we have

$$
\begin{aligned}
\left|w_{\rho, n}\right|_{p^{*}}^{p} & \leq C \int_{\mathbb{R}^{N}}\left|\nabla w_{\rho, n}\right|^{p} d x=C \int_{\mathbb{R}^{N}}\left|\nabla\left(\eta u_{n} u_{\rho, n}^{\sigma-1}\right)\right|^{p} d x \\
& \leq C \sigma^{p}\left(\int_{\mathbb{R}^{N}}|\nabla \eta|^{p} u_{n}^{p} u_{\rho, n}^{p(\sigma-1)} d x+\int_{\mathbb{R}^{N}} \eta^{p} u_{\rho, n}^{p(\sigma-1)}\left|\nabla u_{n}\right|^{p} d x\right) .
\end{aligned}
$$

Combining (4.18)-(4.19), we have

$$
\left|w_{\rho, n}\right|_{p^{*}}^{p} \leq C \sigma^{p}\left(\int_{\mathbb{R}^{N}}|\nabla \eta|^{p}\left|u_{n}\right|^{p} u_{\rho, n}^{p(\sigma-1)} d x+\int_{\mathbb{R}^{N}} u_{n}^{p^{*}} \eta^{p} u_{\rho, n}^{p(\sigma-1)} d x\right) .
$$

Choosing $\sigma=p^{*} / p$, by the definition of $w_{\rho, n}$ and (4.20), we rewrite the last inequality as

$$
\begin{aligned}
\left(\int_{\mathbb{R}^{N}}\right. & \left.\left(\eta u_{n} u_{\rho, n}^{\left(p^{*}-p\right) / p}\right)^{p^{*}} d x\right)^{p / p^{*}} \\
\leq & C(N, p)\left\{\left[\int_{\mathbb{R}^{N}}\left(\eta u_{n} u_{\rho, n}^{\left(p^{*}-p\right) / p}\right)^{p^{*}} d x\right]^{p / p^{*}}\left(\int_{|x|>R-r} u_{n}^{p^{*}} d x\right)^{p^{*}-p / p}\right. \\
& \left.+\int_{\mathbb{R}^{N}}|\nabla \eta|^{p}\left|u_{n}\right|^{p} u_{\rho, n}^{p^{*}-p} d x\right\} \\
\leq & C(N, p)\left\{\left[\int_{\mathbb{R}^{N}}\left(\eta u_{n} u_{\rho, n}^{\left(p^{*}-p\right) / p}\right)^{p^{*}} d x\right]^{p / p^{*}}\left\|u_{n}\right\|_{p^{*}(|x| \geq R / 2)}^{p^{*}-p}\right. \\
& \left.+\int_{\mathbb{R}^{N}}|\nabla \eta|^{p}\left|u_{n}\right|^{p} u_{\rho, n}^{p^{*}-p} d x\right\} .
\end{aligned}
$$

In view of $u_{n} \rightarrow u$ in $W^{1, p}\left(\mathbb{R}^{N}\right)$, for $R$ large enough, we conclude that

$$
\left\|u_{n}\right\|_{p^{*}(|x| \geq R / 2)}^{p^{*}-p} \leq \frac{1}{C(N, p)} \quad \text { uniformly in } n
$$

Hence we obtain

$$
\begin{aligned}
&\left(\int_{|x| \geq R}\left(u_{n} u_{\rho, n}^{\left(p^{*}-p\right) / p}\right)^{p^{*}} d x\right)^{p / p^{*}} \leq\left(\int_{\mathbb{R}^{N}}\left(\eta u_{n} u_{\rho, n}^{\left(p^{*}-p\right) / p}\right)^{p^{*}} d x\right)^{p / p^{*}} \\
& \leq C(N, p) \int_{\mathbb{R}^{N}}|\nabla \eta|^{p}\left|u_{n}\right|^{p} u_{\rho, n}^{p^{*}-p} d x \leq \frac{C}{r^{p}} \int_{\mathbb{R}^{N}}\left|u_{n}\right|^{p^{*}} d x
\end{aligned}
$$

Using Fatou's lemma in the variable $\rho$, we have

$$
u_{n} \in L^{{p^{*}}^{2} / p}(|x| \geq R) \text { for } R \text { large enough. }
$$

Next, if we put $\sigma=p^{*}(t-1) /(p t)$ with $t=p^{*^{2}} /\left(\left(p^{*}-p\right) p\right)$, then $\sigma>1$, $p t /(t-1)<p^{*}$. Now suppose that $u_{n} \in L^{p \sigma t /(t-1)}(|x| \geq R-r)$ for some $\sigma \geq 1$. Returning to (4.20) and using the Hölder inequality with exponent $t /(t-1)$ 
and $t$, we have

$$
\begin{aligned}
\left|w_{\rho, n}\right|_{p^{*}}^{p} \leq & C \sigma^{p}\left\{\left[\int_{|x| \geq R-r}\left(\eta^{p} u_{n}^{p \sigma}\right)^{t /(t-1)} d x\right]^{1-1 / t}\right. \\
& \cdot\left(\int_{|x|>R-r} u_{n}^{\left(p^{*}-p\right) t} d x\right)^{1 / t} \\
& \left.+\frac{\left[R^{N}-(R-r)^{N}\right]^{1 / t}}{r^{p}}\left(\int_{|x| \geq R-r} u_{n}^{p \sigma t /(t-1)} d x\right)^{1-1 / t}\right\} \\
\leq & C \sigma^{p}\left(1+\frac{R^{N / t}}{r^{p}}\right)\left(\int_{|x| \geq R-r} u_{n}^{p \sigma t /(t-1)} d x\right)^{1-1 / t} \cdot
\end{aligned}
$$

Letting $\rho \rightarrow+\infty$ in (4.22), we obtain

$$
\left\|u_{n}\right\|_{p^{*} \sigma(|x| \geq R)}^{p \sigma} \leq C \sigma^{p}\left(1+\frac{R^{N / t}}{r^{p}}\right)\left\|u_{n}\right\|_{p \sigma t /(t-1)(|x| \geq R-r)}^{p \sigma} .
$$

If we set $\chi:=p^{*}(t-1) /(p t), s:=p t /(t-1)$, then

$$
\left\|u_{n}\right\|_{\sigma \chi s,(|x| \geq R)} \leq C^{1 / \sigma} \sigma^{1 / \sigma}\left(1+\frac{R^{N / t}}{r^{p}}\right)^{1 /(p \sigma)}\left\|u_{n}\right\|_{\sigma s(|x| \geq R-r)} .
$$

Let $\sigma=\chi^{m}(m=1,2, \ldots)$, we obtain

$$
\left\|u_{n}\right\|_{\chi^{m+1} s(|x| \geq R)} \leq C^{\chi^{-m}} \chi^{m \chi^{-m}}\left(1+\frac{R^{N / t}}{r^{p}}\right)^{1 /(p \sigma)}\left\|u_{n}\right\|_{\chi^{m} s(|x| \geq R-r)} .
$$

It is clear that $p>N / t$. Therefore, if we take $r_{m}:=2^{-(m+1)} R$, then inequality (4.23) implies

$$
\begin{aligned}
\left\|u_{n}\right\|_{\chi^{m+1} s(|x| \geq R)} & \leq\left|u_{n}\right|_{\chi^{m+1} s\left(|x| \geq R-r_{m+1}\right)} \\
& \leq C^{\sum_{i=1}^{m} \chi^{-i} \sum^{i=1} \chi^{m} i \chi^{-i}} \exp \left(\sum_{i=1}^{m} \frac{\ln \left(1+2^{p(i+1)}\right)}{p \chi^{i}}\right)\left\|u_{n}\right\|_{\chi s,\left(|x| \geq R-r_{1}\right)} \\
& \leq C\left\|u_{n}\right\|_{p^{*}(|x| \geq R / 2)} .
\end{aligned}
$$

Letting $m \rightarrow \infty$ in the last inequality, we get $\left\|u_{n}\right\|_{L^{\infty}(|x| \geq R)} \leq C\left|u_{n}\right|_{p^{*}(|x| \geq R / 2)}$. By the convergence of $\left\{u_{n}\right\}$ to $u$ in $W^{1, p}\left(\mathbb{R}^{N}\right)$, for any fixed $a>0$, there exists $R>0$ such that $\left\|u_{n}\right\|_{L^{\infty}(|x| \geq R)} \leq a$ for all $n \in \mathbb{N}$. Therefore, $\lim _{|x| \rightarrow \infty} u_{n}(x)=0$ uniformly in $n$.

\section{The existence of multi-bump positive solutions}

For $j \in \Gamma$, we consider the following two functionals:

$$
\begin{aligned}
I_{j}(u):=\frac{1}{p} \int_{\Omega_{j}}\left(|\nabla u|^{p}+Z(x)|u|^{p}\right) d x & \\
& +\frac{b}{2}\left(\int_{\Omega_{j}} \frac{1}{p}|\nabla u|^{p} d x\right)^{2}-\alpha \int_{\Omega_{j}} F(u) d x-\frac{1}{p^{*}} \int_{\Omega_{j}}\left(u_{+}\right)^{p^{*}} d x
\end{aligned}
$$


and

$$
\begin{aligned}
\Phi_{\lambda, j}(u):=\frac{1}{p} \int_{\Omega_{j}^{\prime}}\left(|\nabla u|^{p}+(\lambda V(x)+Z(x))|u|^{p}\right) d x \\
\quad+\frac{b}{2}\left(\int_{\Omega_{j}^{\prime}} \frac{1}{p}|\nabla u|^{p} d x\right)^{2}-\alpha \int_{\Omega_{j}^{\prime}} F(u) d x-\frac{1}{p^{*}} \int_{\Omega_{j}^{\prime}}\left(u_{+}\right)^{p^{*}} d x .
\end{aligned}
$$

We know that the critical points of the above functions are related with the weak solutions to the problem

$$
\begin{cases}-\left(1+b \int_{\mathbb{R}^{N}}|\nabla u|^{p} d x\right) \Delta_{p} u+Z(x) u^{p-1}=\alpha f(u)+u^{p^{*}-1} & \text { in } \Omega_{j} \\ u=0 & \text { on } \partial \Omega_{j}\end{cases}
$$

and

$$
\begin{cases}-\left(1+b \int_{\mathbb{R}^{N}}|\nabla u|^{p} d x\right) \Delta_{p} u+(\lambda V(x)+Z(x)) u^{p-1} & \\ =\alpha f(u)+u^{p^{*}-1} & \text { in } \Omega_{j}^{\prime}, \\ \frac{\partial u}{\partial \eta}=0 & \text { on } \partial \Omega_{j}^{\prime} .\end{cases}
$$

It is easy to check that functionals $I_{j}$ and $\Phi_{\lambda, j}$ satisfy the mountain pass geometry. That is,

(i) $I_{j}(0)=\Phi_{\lambda, j}(0)=0$.

(ii) There exist $\rho_{0}>0$ and $\rho_{1}$ independent of $\lambda \geq 0$ such that

$$
\begin{aligned}
& \|u\|_{0, \Omega_{j}} \leq \rho_{0} \Rightarrow I_{j}(u) \geq 0, \\
& \|u\|_{0, \Omega_{j}}=\rho_{0} \Rightarrow I_{j}(u) \geq \rho_{1}, \\
& \|u\|_{0, \Omega_{j}} \leq \rho_{0} \Rightarrow \Phi_{\lambda, j}(u) \geq 0, \\
& \|u\|_{0, \Omega_{j}}=\rho_{0} \Rightarrow \Phi_{\lambda, j}(u) \geq \rho_{1} .
\end{aligned}
$$

Here we use the notation:

$$
\|u\|_{0, \Omega_{j}}=\int_{\Omega_{j}}\left(|\nabla u|^{p}+Z(x)|u|^{p}\right) d x \quad \text { for } u \in W_{0}^{1, p}\left(\Omega_{j}\right) .
$$

(iii) There exists $\varphi_{j}(x) \in C_{0}^{\infty}\left(\Omega_{j}\right)$ such that

$$
\left\|\varphi_{j}(x)\right\|_{\lambda, \Omega_{j}^{\prime}}=\left\|\varphi_{j}(x)\right\|_{0, \Omega_{j}} \geq \rho_{1}, \quad \Phi_{\lambda, \Omega_{j}^{\prime}}\left(\varphi_{j}\right)=I_{j}\left(\varphi_{j}\right)<0 .
$$

In what follows, we denote by $c_{j}$ and $c_{\lambda, j}$ the minimax level related to the above functions defined by

$$
c_{j}:=\inf _{\gamma \in \Lambda_{j}} \max _{t \in[0,1]} I_{j}(\gamma(t)) \quad \text { and } \quad c_{\lambda, j}:=\inf _{\gamma \in \Lambda_{\lambda, j}} \max _{t \in[0,1]} \Phi_{\lambda, j}(\gamma(t)),
$$

where

$$
\begin{aligned}
\Lambda_{j} & :=\left\{\gamma \in C\left([0,1], W_{0}^{1, p}\left(\Omega_{j}\right)\right): \gamma(0)=0, I_{j}(\gamma(1))<0\right\}, \\
\Lambda_{\lambda, j} & :=\left\{\gamma \in C\left([0,1], W_{0}^{1, p}\left(\Omega_{j}^{\prime}\right)\right): \gamma(0)=0, \Phi_{\lambda, j}(\gamma(1))<0\right\} .
\end{aligned}
$$




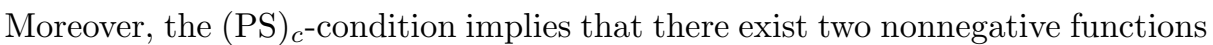
$w_{j} \in W_{0}^{1, p}\left(\Omega_{j}\right)$ and $w_{\lambda, j} \in W_{0}^{1, p}\left(\Omega_{j}^{\prime}\right)$ satisfying

$$
\begin{aligned}
& I_{j}\left(w_{j}\right)=c_{j} \quad \text { and } \quad I_{j}^{\prime}\left(w_{j}\right)=0, \\
& \Phi_{\lambda, j}\left(w_{\lambda, j}\right)=c_{\lambda, j} \quad \text { and } \quad \Phi_{\lambda, j}^{\prime}\left(w_{\lambda, j}\right)=0 .
\end{aligned}
$$

We have the following lemma.

\section{LEMMA 5.1.}

(a) $0<\rho_{1} \leq c_{\lambda, j} \leq c_{j}$ for all $\lambda \geq 0$.

(b) $c_{j}\left(c_{\lambda, j}\right.$, respectively $)$ is a least energy level for $I_{j}(u)\left(\Phi_{\lambda, j}(u)\right.$, respectively), that is

$$
\begin{gathered}
c_{j}=\inf \left\{I_{j}(u): u \in W_{0}^{1, p}\left(\Omega_{j}\right) \backslash\{0\}, I_{j}^{\prime}(u) \cdot u=0\right\}, \\
c_{\lambda, j}=\inf \left\{\Phi_{\lambda, j}(u): u \in W_{0}^{1, p}\left(\Omega_{j}^{\prime}\right) \backslash\{0\}, \Phi_{\lambda, j}^{\prime}(u) \cdot u=0\right\} . \\
\text { (c) } c_{j}=\max _{r>0} I_{j}\left(r w_{j}\right), c_{\lambda, j}=\max _{r>0} I_{\lambda, j}\left(r w_{\lambda, j}\right) .
\end{gathered}
$$

(d) $c_{\lambda, j} \rightarrow c_{j}$ as $j \rightarrow \infty$.

Proof. From (5.7), it is easy to see that $c_{\lambda, j} \geq \rho_{1}$. On the other hand, for any $u \in W_{0}^{1, p}\left(\Omega_{j}\right)$, we may extend $u$ to $\widetilde{u} \in W_{0}^{1, p}\left(\Omega_{j}^{\prime}\right)$ by

$$
\widetilde{u}(x)= \begin{cases}u(x) & \text { in } \Omega_{j}, \\ 0 & \text { in } \Omega_{j}^{\prime} \backslash \bar{\Omega}_{j},\end{cases}
$$

we regard $W_{0}^{1, p}\left(\Omega_{j}\right) \subset W_{0}^{1, p}\left(\Omega_{j}^{\prime}\right)$. Thus we have $\Lambda_{j} \subset \Lambda_{\lambda, j}$ and

$$
\begin{aligned}
c_{\lambda, j} & =\inf _{\gamma \in \Lambda_{\lambda, j}} \max _{t \in[0,1]} \Phi_{\lambda, j}(\gamma(t)) \\
& \leq \inf _{\gamma \in \Lambda_{j}} \max _{t \in[0,1]} \Phi_{\lambda, j}(\gamma(t)) \leq \inf _{\gamma \in \Lambda_{j}} \max _{t \in[0,1]} I_{j}(\gamma(t))=c_{j} .
\end{aligned}
$$

Thus we have (a).

(b)-(c) are standard consequences which can be obtained from the monotonicity of the nonlinearity $g(x, s) / s^{p-1}$ (see Chapter 4 in [45]).

Now we show (d). Using Lemma 4.7, we may extract a subsequence $\lambda_{n} \rightarrow \infty$ such that $w_{\lambda, j} \rightarrow u_{0}$ strongly in $W_{0}^{1, p}\left(\Omega_{j}^{\prime}\right)$, where $u_{0} \in W_{0}^{1, p}\left(\Omega_{j}\right)$ is a solution of (5.1) and $\Phi_{\lambda, j}\left(w_{\lambda, j}\right) \rightarrow I_{j}\left(u_{0}\right)$. By the definition of $c_{j}$, we have

$$
\limsup _{\lambda \rightarrow \infty} c_{\lambda, j}=\limsup _{\lambda \rightarrow \infty} \Phi_{\lambda, j}\left(w_{\lambda, j}\right) \geq I_{j}\left(u_{0}\right) \geq c_{j} .
$$

Compare with (5.8), we get (iv) and this completes the proof of Lemma 5.1.

In what follows, let us fix $R>1$ such that

$$
\left|I_{j}\left(\frac{1}{R} w_{j}\right)\right|<\frac{1}{2} c_{j} \quad \text { for all } j \in \Gamma
$$

and

$$
\left|I_{j}\left(R w_{j}\right)-c_{j}\right| \geq 1 \quad \text { for all } j \in \Gamma \text {. }
$$


From the definition of $c_{j}$, the equality below is standard:

$$
\max _{s \in\left[1 / R^{2}, 1\right]} I_{j}\left(s R w_{j}\right)=c_{j} \quad \text { for all } j \in \Gamma,
$$

where the interval $\left[1 / R^{2}, 1\right]$ is chosen conveniently for our purposes.

Reordering the set $\Gamma$, we may consider $\Gamma=\{1, \ldots, l\}(l \leq k)$,

$$
\begin{gathered}
\gamma_{0}\left(s_{1}, \ldots, s_{l}\right)(x)=\sum_{j=1}^{l} s_{j} R w_{j}(x) \quad \text { for all }\left(s_{1}, \ldots, s_{l}\right) \in\left[\frac{1}{R^{2}}, 1\right]^{l}, \\
\Lambda_{*}:=\left\{\gamma \in C\left(\left[\frac{1}{R^{2}}, 1\right]^{l}, E_{\lambda} \backslash\{0\}\right): \gamma=\gamma_{0} \text { on } \partial\left(\left[\frac{1}{R^{2}}, 1\right]^{l}\right)\right\}
\end{gathered}
$$

and

$$
b_{\lambda, \Gamma}=\inf _{\gamma \in \Lambda_{*}} \max _{\left(s_{1}, \ldots, s_{l}\right) \in\left[1 / R^{2}, 1\right]^{l}} \Phi_{\lambda}\left(\gamma\left(s_{1}, \ldots, s_{l}\right)\right) .
$$

Remark 5.2. As $\gamma_{0} \in \Lambda_{*}, \Lambda_{*} \neq \emptyset$ and $b_{\lambda, \Gamma}$ is well-defined.

Lemma 5.3. For any $\gamma \in \Lambda_{*}$, there exists $\left(t_{1}, \ldots, t_{l}\right) \in\left[1 / R^{2}, 1\right]^{l}$ such that

$$
\Phi_{\lambda, j}^{\prime}\left(\gamma\left(t_{1}, \ldots, t_{l}\right)\right)\left(\gamma\left(t_{1}, \ldots, t_{l}\right)\right)=0 \quad \text { for all } j \in\{1, \ldots, l\} .
$$

Proof. For a given $\gamma \in \Lambda_{*}$, let us consider the map $\widetilde{\gamma}:\left[1 / R^{2}, 1\right]^{l} \rightarrow R^{l}$ defined by

$$
\widetilde{\gamma}\left(s_{1}, \ldots, s_{l}\right)=\left(\Phi_{\lambda, 1}^{\prime}(\gamma)(\gamma), \ldots, \Phi_{\lambda, l}^{\prime}(\gamma)(\gamma)\right),
$$

where $\Phi_{\lambda, j}^{\prime}(\gamma)(\gamma)=\Phi_{\lambda, j}^{\prime}\left(\gamma\left(s_{1}, \ldots, s_{l}\right)\right) \gamma\left(s_{1}, \ldots, s_{l}\right)$ for all $j \in \Gamma$.

For any $\left(s_{1}, \ldots, s_{l}\right) \in \partial\left(\left[1 / R^{2}, 1\right]^{l}\right)$, it follows that

$$
\begin{gathered}
\gamma\left(s_{1}, \ldots, s_{l}\right)=\gamma_{0}\left(s_{1}, \ldots, s_{l}\right), \\
\Phi_{\lambda, j}^{\prime}\left(\gamma_{0}\left(s_{1}, \ldots, s_{l}\right)\right) \gamma_{0}\left(s_{1}, \ldots, s_{l}\right)=0 .
\end{gathered}
$$

From this fact, we have $s_{j} \notin\left\{1 / R^{2}, 1\right\}$ for $j \in \Gamma$. Thus $(0, \ldots, 0) \notin \widetilde{\gamma}\left(\partial\left(\left[1 / R^{2}, 1\right]^{l}\right)\right)$. Hence, it follows from the topological degree that

$$
\operatorname{deg}\left(\widetilde{\gamma},\left(\frac{1}{R^{2}}, 1\right)^{l},(0, \ldots, 0)\right)=(-1)^{l} \neq 0 .
$$

Thus, there exists $\left(t_{1}, \ldots, t_{l}\right) \in\left(1 / R^{2}, 1\right)^{l}$ satisfying

$$
\Phi_{\lambda, j}^{\prime}\left(\gamma\left(t_{1}, \ldots, t_{l}\right)\right)\left(\gamma\left(t_{1}, \ldots, t_{l}\right)\right)=0 \text { for } j \in\{1, \ldots, l\} .
$$

This completes the proof of Lemma 5.3.

In the sequel, the number $c_{\Gamma}=\sum_{j=1}^{l} c_{j}$ is very important in the proof of Theorem 1.1. Let us analyse the interaction between $\sum_{j=1}^{l} c_{\lambda, j}, b_{\lambda, \Gamma}$ and $c_{\Gamma}$, using the fact that $c_{\Gamma} \in\left(0, S^{N / p} /(2 p)\right)$ (see Remark 4.4). 
LEMMA 5.4. We have the following facts:

(a) $\sum_{j=1}^{l} c_{\lambda, j} \leq b_{\lambda, \Gamma} \leq c_{\Gamma}$ for all $\lambda \geq 1$.

(b) $\Phi_{\lambda}\left(\gamma\left(s_{1}, \ldots, s_{l}\right)\right)<c_{\Gamma}$ for all $\lambda \geq 1, \gamma \in \Lambda_{*}$ and $\left(s_{1}, \ldots, s_{l}\right) \in \partial\left(\left[1 / R^{2}, 1\right]^{l}\right)$.

Proof. Since $\gamma_{0}$ defined in (5.10) belongs to $\Lambda_{*}$, we have

$$
\begin{aligned}
b_{\lambda, \Gamma} & \leq \max _{\left(s_{1}, \ldots, s_{l}\right) \in\left[1 / R^{2}, 1\right]^{l}} \Phi_{\lambda}\left(\gamma_{0}\left(s_{1}, \ldots, s_{l}\right)\right) \\
& =\max _{\left(s_{1}, \ldots, s_{l}\right) \in\left[1 / R^{2}, 1\right]^{l}} \sum_{j=1}^{l} I_{j}\left(s R w_{j}\right)=\sum_{j=1}^{l} c_{j}=c_{\Gamma} .
\end{aligned}
$$

Fix $\left(t_{1}, \ldots, t_{l}\right) \in\left[1 / R^{2}, 1\right]^{l}$ given in Lemma 5.3 and recall that $c_{\lambda, j}$ is given by

$$
c_{\lambda, j}=\inf \left\{\Phi_{\lambda, j}(u): u \in W_{0}^{1, p}\left(\Omega_{j}^{\prime}\right) \backslash\{0\}, \Phi_{\lambda, j}^{\prime}(u) \cdot u=0\right\} .
$$

It follows that $\Phi_{\lambda, j}\left(\gamma\left(t_{1}, \ldots, t_{l}\right)\right) \geq c_{\lambda, j}$ for all $j \in \Gamma$. On the other hand,

$$
\Phi_{\lambda}(u)=\Phi_{\lambda, \mathbb{R}^{N} \backslash \Omega_{\Gamma}^{\prime}}(u)+\sum_{j=1}^{l} \Phi_{\lambda, \Omega_{j}^{\prime}}(u),
$$

where

$$
\begin{aligned}
\Phi_{\lambda, \mathbb{R}^{N} \backslash \Omega_{\Gamma}^{\prime}}(u):= & \frac{1}{p} \int_{\lambda, \mathbb{R}^{N} \backslash \Omega_{\Gamma}^{\prime}}\left(|\nabla u|^{p}+(\lambda V(x)+Z(x))|u|^{p}\right) d x \\
& +\frac{b}{2}\left(\int_{\lambda, \mathbb{R}^{N} \backslash \Omega_{\Gamma}^{\prime}} \frac{1}{p}|\nabla u|^{p} d x\right)^{2}-\int_{\lambda, \mathbb{R}^{N} \backslash \Omega_{\Gamma}^{\prime}} \widetilde{F}(u) d x .
\end{aligned}
$$

If we recall the definition of $\widetilde{F}(u)$, we have $\widetilde{F}(u) \leq \nu_{0}|s|^{p} / p$. This fact together with Lemma 2.1 imply that

$$
\begin{aligned}
\Phi_{\lambda, \mathbb{R}^{N} \backslash \Omega_{\Gamma}^{\prime}}(u):= & \frac{1}{p} \int_{\lambda, \mathbb{R}^{N} \backslash \Omega_{\Gamma}^{\prime}}\left(|\nabla u|^{p}+(\lambda V(x)+Z(x))|u|^{p}\right) d x \\
& +\frac{b}{2}\left(\int_{\lambda, \mathbb{R}^{N} \backslash \Omega_{\Gamma}^{\prime}} \frac{1}{p}|\nabla u|^{p} d x\right)^{2}-\int_{\lambda, \mathbb{R}^{N} \backslash \Omega_{\Gamma}^{\prime}} \widetilde{F}(u) d x \\
\geq & \frac{1}{p}\left(\|u\|_{\lambda, \mathbb{R}^{N} \backslash \Omega_{\Gamma}^{\prime}}^{p}-\nu_{0}|u|_{p, \mathbb{R}^{N} \backslash \Omega_{\Gamma}^{\prime}}^{p} \geq \frac{1}{p} \delta_{0}\|u\|_{\lambda, \mathbb{R}^{N} \backslash \Omega_{\Gamma}^{\prime}}^{p} \geq 0 .\right.
\end{aligned}
$$

Thus

$$
\begin{aligned}
\Phi_{\lambda}(u) & =\Phi_{\lambda, \mathbb{R}^{N} \backslash \Omega_{\Gamma}^{\prime}}(u)+\sum_{j=1}^{l} \Phi_{\lambda, \Omega_{j}^{\prime}}(u) \geq \sum_{j=1}^{l} \Phi_{\lambda, \Omega_{j}^{\prime}}(u) \\
& \geq \sum_{j=1}^{l} \inf \left\{\Phi_{\lambda, j}(u): u \in E_{\lambda} \backslash\{0\}, \Phi_{\lambda, j}^{\prime}(u) \cdot u=0\right\}=\sum_{j=1}^{l} c_{\lambda, j} .
\end{aligned}
$$


From definition of $b_{\lambda, \Gamma}$, we conclude that

$$
b_{\lambda, \Gamma} \geq \sum_{j=1}^{l} c_{\lambda, j} .
$$

This completes the proof of Lemma 5.4 (a).

Since $\gamma\left(s_{1}, \ldots, s_{l}\right)=\gamma_{0}\left(s_{1}, \ldots, s_{l}\right)$ on $\partial\left(\left[1 / R^{2}, 1\right]^{l}\right)$ we have

$$
\Phi_{\lambda}\left(\gamma_{0}\left(s_{1}, \ldots, s_{l}\right)\right)=\sum_{j=1}^{l} I_{j}\left(s_{j} R w_{j}\right) .
$$

Moreover, $I_{j}\left(s_{j} R w_{j}\right) \leq c_{j}$ for all $j \in \Gamma$ and for some $j_{0} \in \Gamma, s_{j_{0}} \in\left\{1 / R^{2}, 1\right\}$ and $I_{j_{0}}\left(s_{j_{0}} R w_{j 0}\right) \leq c_{j_{0}} / 2$. Therefore, $\Phi_{\lambda}\left(\gamma_{0}\left(s_{1}, \ldots, s_{l}\right)\right) \leq c_{\Gamma}-\varepsilon$, for some $\varepsilon>0$. This completes the proof of Lemma $5.4(\mathrm{~b})$.

COROLlary 5.5.

(a) $b_{\lambda, \Gamma} \rightarrow c_{\Gamma}$ as $\lambda \rightarrow \infty$.

(b) $b_{\lambda, j}$ is a critical value of $\Phi_{\lambda}$ for large $\lambda$.

Proof. (a) For all $\lambda \geq 1$ and for each $j$ we have $0<c_{\lambda, j} \leq c_{j}$. Using the same type of idea as explored in the proof of Lemma 4.7, we can prove that $c_{\lambda, j} \rightarrow c_{j}$ as $\lambda \rightarrow \infty$ and thus, from Lemma $5.4, b_{\lambda, \Gamma} \rightarrow c_{\Gamma}$ as $\lambda \rightarrow \infty$.

(b) By Corollary 5.5 (a) and (4.5), we may choose $\lambda$ large enough such that $b_{\lambda, \Gamma}, c_{\Gamma} \in\left(0, S^{N / p} /(2 p)\right)$. Lemma 4.5 implies that any $(\mathrm{PS})_{b_{\lambda, \Gamma} \text {-sequence of }}$ the functional $\Phi_{\lambda}$ has a strongly convergent subsequence in $E_{\lambda}$. Employing this fact, we can use the well-known arguments involving the deformation lemma to conclude that $b_{\lambda, \Gamma}$ is a critical level of $\Phi_{\lambda}$ for $\lambda \geq 1$.

In order to prove Theorem 1.1, we need to find a positive solution $u_{\lambda}$ for $\lambda$ large enough, which approaches a least-energy solution in each $\Omega_{j}(j \in \Gamma)$ and vanishes elsewhere as $\lambda \rightarrow \infty$. To this end, we will prove two lemmas that, together with the estimates made in the above section, imply that Theorem 1.1 holds.

Hereafter

$$
M:=1+\sum_{j=1}^{k} \sqrt{\left(\frac{1}{p}-\frac{1}{\theta}\right)^{-1} c_{j}}, \quad \bar{B}_{M+1}(0):=\left\{u \in E_{\lambda}:\|u\|_{\lambda} \leq M+1\right\}
$$

and we choose

$$
0<\mu<\frac{1}{3} \min _{j \in\{1, \ldots, l\}} c_{j}
$$

We define

$$
D_{\lambda}^{\mu}:=\left\{u \in \bar{B}_{M+1}(0):\|u\|_{\lambda, \mathbb{R}^{N} \backslash \Omega_{j}^{\prime}} \leq \mu,\left|\Phi_{\lambda, \Omega_{j}^{\prime}}(u)-c_{j}\right| \leq \mu \text { for all } j \in \Gamma\right\} .
$$


We also use the notation $\Phi_{\lambda}^{c_{\Gamma}}:=\left\{u \in E_{\lambda}: \Phi_{\lambda}(u) \leq c_{\Gamma}\right\}$. We remark that $D_{\mu}^{\lambda} \cap \Phi_{\lambda}^{c_{\Gamma}}$ contains all functions of the form

$$
w(x)= \begin{cases}w_{j}(x) & \text { in } \Omega_{j}, \\ 0 & \text { in } \mathbb{R}^{N} \backslash \bar{\Omega}_{\Gamma} .\end{cases}
$$

We have the following lemma.

Lemma 5.6. There exist $\sigma_{0}>0$ and $\Lambda_{0} \geq 0$ independent of $\lambda$ such that

$$
\left\|\Phi_{\lambda}^{\prime}(u)\right\|_{\lambda} \geq \sigma_{0} \quad \text { for } \lambda_{0} \geq \Lambda_{0} \text { and for all } u \in\left(D_{\lambda}^{2 \mu} \backslash D_{\lambda}^{\mu}\right) \cap \Phi_{\lambda}^{c_{\Gamma}} .
$$

Proof. We prove it by contradiction. Suppose that there exist $\lambda_{n} \rightarrow \infty$ and $u_{n} \in\left(D_{\lambda}^{2 \mu} \backslash D_{\lambda}^{\mu}\right) \cap \Phi_{\lambda}^{c_{\Gamma}}$ such that $\left\|\Phi_{\lambda}^{\prime}\left(u_{n}\right)\right\|_{\lambda} \rightarrow 0$. Since $u_{n} \in D_{\lambda_{n}}^{2 \mu}$, thus $u_{n}$ is bounded in $E_{\lambda}$ and $\Phi_{\lambda_{n}}\left(u_{n}\right)$ stays bounded as $n \rightarrow \infty$. We may assume that $\Phi_{\lambda_{n}}\left(u_{n}\right) \rightarrow c \leq c_{\Gamma}$ up to a subsequence.

Applying Lemma 4.7, we can extract a subsequence $u_{n} \rightarrow u$ in $W^{1, p}\left(\mathbb{R}^{N}\right)$ and

$$
\begin{gathered}
\lim _{n \rightarrow \infty} \Phi_{\lambda_{n}}\left(u_{n}\right)=\sum_{j=1}^{l} I_{j}(u) \leq c_{\Gamma}, \\
u_{n} \rightarrow u \quad \text { in } W^{1, p}\left(\mathbb{R}^{N}\right), \\
\int_{\mathbb{R}^{N}} \lambda_{n} V(x)\left|u_{n}\right|^{p} d x \rightarrow 0, \\
\left\|u_{n}\right\|_{\lambda_{n}, \mathbb{R}^{N} \backslash \Omega_{\Gamma}} \rightarrow 0 .
\end{gathered}
$$

Since $c_{\Gamma}=\sum_{j=1}^{l} c_{j}$ and $c_{j}$ is the least energy level for $I_{j}(u)$, we have the following two possibilities:

(1) $I_{j}\left(\left.u\right|_{\Omega_{j}}\right)=c_{j}$ for all $j \in \Gamma$.

(2) $I_{j_{0}}\left(\left.u\right|_{\Omega_{j_{0}}}\right)=0$, that is $\left.u\right|_{\Omega_{j_{0}}}=0$ for some $j_{0} \in \Gamma$.

If (1) occurs, we have

$$
\begin{aligned}
\frac{1}{p} \int_{\Omega_{j}}\left(|\nabla u|^{p}+Z(x)|u|^{p}\right) d x+\frac{b}{2}\left(\int_{\Omega_{j}} \frac{1}{p}|\nabla u|^{p} d x\right)^{2} & \\
& \quad-\alpha \int_{\Omega_{j}} f(u) d x-\frac{1}{p^{*}} \int_{\Omega_{j}} u^{p^{*}} d x=c_{j}
\end{aligned}
$$

for all $j \in \Gamma$. It follows from (5.13)-(5.16) and together with the fact that $u_{n} \in D_{\lambda_{n}}^{\mu}$ for large $n$ which is a contradiction to $u_{n} \in\left(D_{\lambda}^{2 \mu} \backslash D_{\lambda}^{\mu}\right)$.

If (2) occurs, from (5.14) and (5.15) it follows that $\left|\Phi_{\lambda, j}\left(u_{n}\right)-c_{j_{0}}\right| \rightarrow c_{j_{0}} \geq 3 \mu$. This is also a contraction to $u_{n} \in\left(D_{\lambda}^{2 \mu} \backslash D_{\lambda}^{\mu}\right)$ and we complete the proof.

The following lemma is the key of the proof of our main result. 
LEMma 5.7. Let $\mu$ satisfy (5.11) and let $\Lambda_{0}$ be the constant given in Lemma 5.6. Then for any $\lambda \geq \Lambda_{0}$, there exists a solution $u_{\lambda}$ of problem (3.3) satisfying $u_{\lambda} \in D_{\lambda}^{\mu} \cap \Phi_{\lambda}^{c_{\Gamma}}$.

Proof. Assume by contradiction that there are no critical points in $D_{\lambda}^{\mu} \cap \Phi_{\lambda}^{c_{\Gamma}}$. Since the Palais-Smale condition holds for $\Phi_{\lambda}(u)$ in $\left(0,(a S)^{N / p} /(2 p)\right)$, there exists a constant $d_{\lambda}>0$ such that $\left\|\Phi_{\lambda}^{\prime}(u)\right\| \geq d_{\lambda}$ for all $u \in D_{\lambda}^{\mu} \cap \Phi_{\lambda}^{c_{\Gamma}}$ and, from Lemma 5.6, we have $\left\|\Phi_{\lambda}^{\prime}(u)\right\| \geq \sigma_{0}$ for all $u \in\left(D_{\lambda}^{2 \mu} \backslash D_{\lambda}^{\mu}\right) \cap \Phi_{\lambda}^{c_{\Gamma}}$.

Let $\varphi: E_{\lambda} \rightarrow \mathbb{R}$ be a Lipschitz continuous function such that

$$
\varphi(u)= \begin{cases}1 & \text { for } u \in D_{\lambda}^{3 \mu / 2}, \\ 0 & \text { for } u \notin D_{\lambda}^{2 \mu},\end{cases}
$$

and $0 \leq \varphi(u) \leq 1$ for any $u \in E_{\lambda}$. For any $u \in \Phi_{\lambda}^{c_{\Gamma}}$, we define

$$
W(u):=-\varphi(u) \frac{\Phi_{\lambda}^{\prime}(u)}{\left\|\Phi_{\lambda}^{\prime}(u)\right\|_{\lambda}}: \Phi_{\lambda}^{c_{\Gamma}} \rightarrow E_{\lambda} .
$$

Here we identify $E_{\lambda}^{*}$ and $E_{\lambda}$ by the Riesz representation theorem. We consider the deformation flow $\eta:[0, \infty) \times \Phi_{\lambda}^{c_{\Gamma}} \rightarrow \Phi_{\lambda}^{c_{\Gamma}}$ defined by

$$
\frac{d \eta}{d t}=W(\eta(t, u)), \quad \eta(0, u)=u \in \Phi_{\lambda}^{c_{\Gamma}} .
$$

Thus $\eta$ has the following properties:

$$
\begin{gathered}
\frac{d}{d t} \Phi_{\lambda}(\eta(t, u))=-\varphi(\eta(t, u))\left\|\Phi_{\lambda}^{\prime}(\eta(t, u))\right\|_{\lambda} \leq 0 \\
\left\|\frac{d \eta}{d t}\right\|_{\lambda} \leq 1 \text { for all } t, u \\
\eta(t, u)=u \text { for all } t \geq 0 \text { and } u \in \Phi_{\lambda}^{c_{\Gamma}} \backslash D_{\lambda}^{2 \mu} .
\end{gathered}
$$

Let $\gamma_{0}\left(s_{1}, \ldots, s_{l}\right) \in \Lambda_{*}$ be a path defined in (5.10). We consider $\eta\left(t, \gamma_{0}\left(s_{1}, \ldots, s_{l}\right)\right)$ for large enough $t$. Since for all $\left(s_{1}, \ldots, s_{l}\right) \in \partial\left(\left[1 / R^{2}, 1\right]^{l}\right), \gamma_{0}\left(s_{1}, \ldots, s_{l}\right) \notin D_{\lambda}^{2 \mu}$, thus we have by (5.19) that

$$
\eta\left(t, \gamma_{0}\left(s_{1}, \ldots, s_{l}\right)\right)=\gamma_{0}\left(s_{1}, \ldots, s_{l}\right) \quad \text { for all }\left(s_{1}, \ldots, s_{l}\right) \in \partial\left(\left[1 / R^{2}, 1\right]^{l}\right)
$$

and $\eta\left(t, \gamma_{0}\left(s_{1}, \ldots, s_{l}\right)\right) \in \Lambda_{*}$ for all $t \geq 0$.

Since supp $\gamma_{0}\left(\left(s_{1}, \ldots, s_{l}\right)(x)\right) \subset \bar{\Omega}_{\Gamma}$ for all $\left(s_{1}, \ldots, s_{l}\right) \in \partial\left(\left[1 / R^{2}, 1\right]^{l}\right)$ and hence $\Phi_{\lambda}\left(\gamma_{0}\left(s_{1}, \ldots, s_{l}\right)(x)\right)$ and $\left\|\gamma_{0}\left(s_{1}, \ldots, s_{l}\right)(x)\right\|_{\lambda, j}$, etc. do not depend on $\lambda \geq$ 0 . On the other hand, $\Phi_{\lambda}\left(\gamma_{0}\left(s_{1}, \ldots, s_{l}\right)(x)\right) \leq c_{\Gamma}$ for all $\left(s_{1}, \ldots, s_{l}\right) \in\left[1 / R^{2}, 1\right]^{l}$ and $\Phi_{\lambda}\left(\gamma_{0}\left(s_{1}, \ldots, s_{l}\right)(x)\right)=c_{\Gamma}$ if and only if $s_{j}=1 / R$, that is $\left.\gamma_{0}\left(s_{1}, \ldots, s_{l}\right)(x)\right|_{\Omega_{j}}$ $=w_{j}$ for $j \in \Gamma$. Thus we have

$$
m_{0}:=\max \left\{\Phi_{\lambda}(u): u \in \gamma_{0}\left(\left[1 / R^{2}, 1\right]^{l}\right) \backslash D_{\lambda}^{\mu}\right\}
$$

is dependent of $\lambda$ and $m_{0}<c_{\Gamma}$. 
From (5.19), it is easy to see that for any $t>0$

$$
\left\|\eta\left(0, \gamma_{0}\left(s_{1}, \ldots, s_{l}\right)\right)-\eta\left(t, \gamma_{0}\left(s_{1}, \ldots, s_{l}\right)\right)\right\|_{\lambda} \leq t .
$$

Since $\Phi_{\lambda, j}(u) \in C^{1}\left(E_{\lambda}\right)$ for all $j=1, \ldots, l$, and due to assumptions $\left(\mathrm{f}_{1}\right)-\left(\mathrm{f}_{4}\right)$, it is easy to see that for large enough $T$, there exists a positive number $p_{0}>0$ which is independent of $\lambda$ such that for all $j=1, \ldots, l$ and $t \in[0, T]$,

$$
\left\|\Phi_{\lambda, j}^{\prime}\left(\eta\left(t, \gamma_{0}\left(s_{1}, \ldots, s_{l}\right)\right)\right)\right\|_{\lambda} \leq p_{0} .
$$

We claim that for large enough $T$,

$$
\max _{\left(s_{1}, \ldots, s_{l}\right) \in\left[1 / R^{2}, 1\right]^{l}} \Phi_{\lambda}\left(\eta\left(T, \gamma_{0}\left(s_{1}, \ldots, s_{l}\right)(x)\right)\right) \leq \max \left\{m_{0}, c_{\Gamma}-\tau_{0} \mu / 2\right\},
$$

where $m_{0}$ is given in $(5.20), \tau_{0}=\max \left\{\sigma_{0}, \sigma_{0} / p_{0}\right\}$. In fact, if $\gamma_{0}\left(s_{1}, \ldots, s_{l}\right)(x) \notin$ $D_{\lambda}^{\mu}$, then by $(5.21)$ we have $\Phi_{\lambda}\left(\eta\left(T, \gamma_{0}\left(s_{1}, \ldots, s_{l}\right)(x)\right)\right) \leq m_{0}$ and thus (5.22) holds. Now we consider the case $\gamma_{0}\left(s_{1}, \ldots, s_{l}\right)(x) \in D_{\lambda}^{\mu}$, we consider the behavior of $\widetilde{\eta}(t):=\eta\left(t, \gamma_{0}\left(s_{1}, \ldots, s_{l}\right)\right)$.

We set $\widetilde{d}_{\lambda}:=\min \left\{d_{\lambda}, \sigma_{0}\right\}$ and $T=\sigma_{0} \mu /\left(2 \widetilde{d}_{\lambda}\right)$. We consider two cases:

(1) $\widetilde{\eta}(t) \in D_{\lambda}^{3 \mu / 2}$ for all $t \in[0, T]$.

(2) $\widetilde{\eta}\left(t_{0}\right) \in \partial D_{\lambda}^{3 \mu / 2}$ for some $t_{0} \in[0, T]$.

When (1) holds, we have $\varphi(\widetilde{\eta}(t)) \equiv 1$ and $\left\|\Phi_{\lambda}^{\prime}(\widetilde{\eta}(t))\right\|_{\lambda} \geq \widetilde{d}_{\lambda}$ for all $t \in[0, T]$. Thus by (5.17), we have

$$
\begin{aligned}
\Phi_{\lambda}(\widetilde{\eta}(T)) & =\Phi_{\lambda}\left(\gamma_{0}\left(s_{1}, \ldots, s_{l}\right)\right)+\int_{0}^{T} \frac{d}{d s} \Phi_{\lambda}(\widetilde{\eta}(t)) \\
& =\Phi_{\lambda}\left(\gamma_{0}\left(s_{1}, \ldots, s_{l}\right)\right)-\int_{0}^{T} \varphi(\widetilde{\eta}(s))\left\|\Phi_{\lambda}^{\prime}(\widetilde{\eta}(s))\right\|_{\lambda} d s \\
& \leq c_{\Gamma}-\int_{0}^{T} \widetilde{d}_{\lambda} d s=c_{\Gamma}-\widetilde{d}_{\lambda} T=c_{\Gamma}-\frac{1}{2} \sigma_{0} \mu \leq c_{\Gamma}-\frac{1}{2} \tau_{0} \mu .
\end{aligned}
$$

When (2) holds, there exists $0 \leq t_{1}<t_{2} \leq T$ such that

$$
\begin{gathered}
\widetilde{\eta}\left(t_{1}\right) \in \partial D_{\lambda}^{\mu}, \\
\widetilde{\eta}\left(t_{2}\right) \in \partial D_{\lambda}^{3 \mu / 2}, \\
\widetilde{\eta}(t) \in \partial D_{\lambda}^{3 \mu / 2} \backslash D_{\lambda}^{\mu} \quad \text { for all } t \in\left[t_{1}, t_{2}\right] .
\end{gathered}
$$

It follows (5.24) that, for some $j_{0} \in \Gamma$,

$$
\left\|\widetilde{\eta}\left(t_{2}\right)\right\|_{\lambda, \mathbb{R}^{N} \backslash \Omega_{\Gamma}^{\prime}}=\frac{3 \mu}{2} \quad \text { or } \quad\left|\Phi_{\lambda, \Omega_{j_{0}}^{\prime}}\left(\widetilde{\eta}\left(t_{2}\right)\right)-c_{j_{0}}\right|=\frac{3 \mu}{2} .
$$

We only consider the latter case, the former case can be obtained in a similar way. By (5.23), $\left|\Phi_{\lambda, \Omega_{j_{0}}^{\prime}}\left(\widetilde{\eta}\left(t_{1}\right)\right)-c_{j_{0}}\right| \leq \mu$. Thus we have $\left|\Phi_{\lambda, \Omega_{j_{0}}^{\prime}}\left(\widetilde{\eta}\left(t_{2}\right)\right)-\Phi_{\lambda, \Omega_{j_{0}}^{\prime}}\left(\widetilde{\eta}\left(t_{1}\right)\right)\right| \geq\left|\Phi_{\lambda, \Omega_{j_{0}}^{\prime}}\left(\widetilde{\eta}\left(t_{2}\right)\right)-c_{j_{0}}\right|-\left|\Phi_{\lambda, \Omega_{j_{0}}^{\prime}}\left(\widetilde{\eta}\left(t_{1}\right)\right)-c_{j_{0}}\right| \geq \frac{1}{2} \mu$. 
On the other hand, by the mean value theorem, there exists $t^{\prime} \in\left(t_{1}, t_{2}\right)$ such that

$$
\left|\Phi_{\lambda, \Omega_{j_{0}}^{\prime}}\left(\widetilde{\eta}\left(t_{2}\right)\right)-\Phi_{\lambda, \Omega_{j_{0}}^{\prime}}\left(\widetilde{\eta}\left(t_{1}\right)\right)\right|=\left|\Phi_{\lambda, \Omega_{j_{0}}^{\prime}} \cdot \frac{d \widetilde{\eta}}{d t}\left(t^{\prime}\right)\right|\left(t_{2}-t_{1}\right) .
$$

From (5.18) and (5.21) we have that $t_{2}-t_{1} \geq \mu / 2 p_{0}$. Thus we have

$$
\begin{aligned}
\Phi_{\lambda}(\widetilde{\eta}(T)) & =\Phi_{\lambda}\left(\gamma_{0}\left(s_{1}, \ldots, s_{l}\right)\right)+\int_{0}^{T} \frac{d}{d s} \Phi_{\lambda}(\widetilde{\eta}(t)) \\
& =\Phi_{\lambda}\left(\gamma_{0}\left(s_{1}, \ldots, s_{l}\right)\right)-\int_{0}^{T} \varphi(\widetilde{\eta}(s))\left\|\Phi_{\lambda}^{\prime}(\widetilde{\eta}(s))\right\|_{\lambda} d s \\
& \leq c_{\Gamma}-\int_{t_{1}}^{t_{2}} \varphi(\widetilde{\eta}(s))\left\|\Phi_{\lambda}^{\prime}(\widetilde{\eta}(s))\right\|_{\lambda} d s \\
& =c_{\Gamma}-\sigma_{0}\left(t_{2}-t_{1}\right)=c_{\Gamma}-\frac{1}{2} \sigma_{0} \mu \leq c_{\Gamma}-\frac{1}{2} \tau_{0} \mu
\end{aligned}
$$

and so (5.22) is proved. We recall that $\widetilde{\eta}(T)=\eta\left(T, \gamma_{0}\left(s_{1}, \ldots, s_{l}\right)\right) \in \Lambda_{*}$. Thus

$$
b_{\lambda, \Gamma} \leq \Phi_{\lambda}(\widetilde{\eta}(T)) \leq \max \left\{m_{0}, c_{\Gamma}-\tau_{0} \mu / 2\right\} .
$$

However, by Corollary 5.5, we have $b_{\lambda, \Gamma} \rightarrow c_{\Gamma}$. This is a contradiction with (5.26), thus $\Phi_{\lambda}(u)$ has a critical point $u_{\lambda} \in D_{\lambda}^{\mu}$ for large enough $\lambda$ and we have completed the proof of Lemma 5.7.

Now we give the proof of main results.

Proof of Theorem 1.1. From Lemma 5.7 there exists a family $\left(u_{\lambda}\right)$ of positive solutions to problem (3.3) verifying the following properties:

(I) For fixed $\mu>0$ there exists $\Lambda_{0}$ such that $\left\|u_{\lambda}\right\|_{\lambda, \mathbb{R}^{N} \backslash \Omega_{\Gamma}^{\prime}} \leq \mu$ for all $\lambda \geq \Lambda_{0}$. Thus, from the proof of Lemma 4.8, for fixed sufficiently small $\mu$, we conclude that $\left|u_{\lambda}\right|_{\infty, \mathbb{R}^{N} \backslash \Omega_{\Gamma}^{\prime}} \leq a$ for all $\lambda \geq \Lambda_{0}$ showing that $u_{\lambda}$ is a positive solution to problem (1.1).

(II) Fix $\lambda_{n} \rightarrow \infty$ and $\mu_{n} \rightarrow 0$, the sequence $\left(u_{n}\right)$ satisfies

- $\Phi_{\lambda_{n}}^{\prime}\left(u_{\lambda_{n}}\right)=0$ for all $n \in \mathbb{N}$,

- $\left\|u_{\lambda_{n}}\right\|_{\lambda_{n}, \mathbb{R}^{N} \backslash \Omega_{\Gamma}^{\prime}} \rightarrow 0$

- $\Phi_{\lambda_{n}, j}\left(u_{\lambda_{n}}\right) \rightarrow c_{j}$ for all $j \in \Gamma$, and

- $u_{\lambda_{n}} \rightarrow u$ in $W^{1, p}\left(\mathbb{R}^{N}\right)$ with $u \in W_{0}^{1, p}\left(\Omega_{\Gamma}\right)$.

The proof of Theorem 1.1 follows.

\section{REFERENCES}

[1] C.O. Alves, Existence of multi-bump solutions for a class of quasilinear problems, Adv. Nonlinear Stud. 6 (2006), 491-509.

[2] C.O. Alves, P.C. Carrião and O. H. Miyagaki, Nonlinear pertubations of a periodic elliptic problem with critical growth, J. Math. Anal. Appl. 260, (2001), 133-146. 
[3] C.O. Alves, D.C. De Morais Filho and M.A.S. Souto, Radially symmetric solutions for a class of critical exponent elliptic problems in $\mathbb{R}^{N}$, Electron. J. Diferential Equations 1996 (1996), 1-12.

[4] _ Multiplicity of $p$ ositive solutions for a class of problems with critic al growth in $\mathbb{R}^{N}$, Proc. Edinb. Math. Soc. 52 (2009), 1-21.

[5] C.O. Alves And G.M. Figueiredo, On multiplicity and concentration of positive solutions for a class of quasilinear problems with critical exponential growth in $\mathbb{R}^{N}$, J. Differential Equations 246 (2009), 1288-1311.

[6] C.O. Alves And M.A.S. Souto, Multiplicity of positive solutions for a class of problems with critical growth in $\mathbb{R}^{2}$, J. Differential Equations 244 (2008), 1502-1520.

[7] A. Ambrosetti, M. Badiale and S. Cingolani, Semiclassical states of nonlinear Schrödinger equations, Arch. Ration. Mech. Anal. 140 (1997), 285-300.

[8] A. Ambrosetti, A. Malchiodi And S. Secchi, Multiplicity results for some nonlinear Schrödinger equations with potentials, Arch. Ration. Mech. Anal. 159 (2001), 253-271.

[9] A. Arosio and S. Panizzi, On the well-posedness of the Kirchhoff string, Trans. Amer. Math. Soc. 348 (1996), 305-330.

[10] T. Bartsch, A. Pankov And Z.Q. Wang, Nonlinear Schrödinger equations with steep potential well, Commun. Contemp. Math. 3 (2001), 549-569.

[11] T. BARTSCH AND Z.Q. WANG, Existence and multiplicity results for some superlinear elliptic problems in $\mathbb{R}^{N}$, Comm. Partial Differential Equations 20 (1995), 1725-1741.

[12] _ Multiple positive solutions for a nonlinear Schrödinger equation, Z. Angew. Math. Phys. 51 (2000), 366-384.

[13] H. Brezis And E. Lieb, A relation between pointwise convergence of functions and convergence of functionals, Proc. Amer. Math. Soc. 88 (1983), 486-490.

[14] J. ByeOn AND Z.Q. WANG, Standing waves with a critical frequency for nonlinear Schrödinger equations, Arch. Ration. Mech. Anal. 165 (2002), 295-316.

[15] D. CAO AND E.S. NousSAiR, Multi-bump standing waves with a critical frequency for nonlinear Schrödinger equations, J. Differential Equations 203 (2004), 292-312.

[16] C. Chen, Y. Kuo and T. Wu, The Nehari manifold for a Kirchhoff type problem involving sign-changing weight functions, J. Differential Equations 250 (2011), 1876-1908.

[17] S. Cingolani And M. Lazzo, Multiple positive solutions to nonlinear Schrödinger equations with competing potential functions, J. Differential Equations 160 (2000), 118-138.

[18] S. Cingolani and S. Secchi, Semiclassical states for NLS equations with magnetic potentials having polynomial growths, J. Math. Phys. 46 (2005), 19 pp.

[19] F.J.S.A. Corrêa And R.G. Nascimento, On a nonlocal elliptic system of p-Kirchhofftype under Neumann boundary condition, Math. Comput. Modelling 49 (2009), 598-604.

[20] F.J.S.A. CorrêA And G.M. Figueiredo, On a elliptic equation of p-Kirchhoff type via variational methods, Bull. Austral. Math. Soc. 74 (2006), 263-277.

[21] M. Del Pino And P. Felmer, Semi-classical states for nonlinear Schrödinger equations, Ann. Inst. H. Poincaré 15 (1998), 127-149.

[22] _ Multi-peak bound states for nonlinear Schrödinger equations, J. Funct. Anal. 149 (1997), 245-265.

[23] _ Local mountain passes for semilinear elliptic problems in unbounded domains, Calc. Var. Partial Differential Equations 4 (1996), 121-137.

[24] Y.H. Ding And F.H. Lin, Solutions of perturbed Schrödinger equations with critical nonlinearity, Calc. Var. Partial Differential Equations 30 (2007), 231-249. 
[25] Y.H. Ding AND J. C. Wei, Semiclassical states for nonlinear Schrödinger equations with sign-changing potentials, J. Funct. Anal. 251 (2007), 546-572.

[26] Y. Ding And K. TANakA, Multiplicity of positive solutions of a nonlinear Schrödinger equation, Manuscripta Math. 112 (2003), 109-135.

[27] D.G. De Figueiredo And Y.H. Ding, Solutions of a nonlinear Schrödinger equation, Discrete Contin. Dynam. Syst. 8 (2002), 563-584.

[28] A. Floer And A. Weinstein, Nonspreading wave packets for the cubic Schrödinger equation with a bounded potential, J. Funct. Anal. 69 (1986), 397-408.

[29] X. He And W. Zou, Infinitely many positive solutions for Kirchhoff-type problems, Nonlinear Anal. 70 (2009), 1407-1414.

[30] X.M. He And W.M. Zou, Existence and concentration behavior of positive solutions for a Kirchhoff equation in $\mathbb{R}^{3}$, J. Differential Equations 2 (2012), 1813-1834.

[31] C. He AND G. LI, The regularity of weak solutions to nonlinear scalar field elliptic equations containing $p$ and $q$ Laplacians, Annales Academic Scientiarum Fennica Mathematica, Vol. 33 (2008), 337-371.

[32] G. Kirchnoff, Mechanik, Teubner, Leipzig, 1883.

[33] J.L. Lions, On some equations in boundary value problems of mathematical physics, Contemporary Developments in Continuum Mechanics and Partial Differential Equations, Proc. Internat. Sympos., Inst. Mat. Univ. Fed. Rio de Janeiro, Rio de Janeiro, 1977; NorthHolland Math. Stud., vol. 30, North-Holland, Amsterdam, 1978, 284-346.

[34] _ The concentration compactness principle in the calculus of variations. The locally compact case, Parts I and II, Ann. Inst. H. Poincaré Anal. Non. Linéaire. 1 (1984), 109-145 and 223-283.

[35] G. LI, Some properties of weak solutions of nonlinear scalar field equations, Ann. Acad. Sci. Fenn. Math. Ser. A 14 (1989), 27-36.

[36] S. LIANG AND S. SHI, Existence of multi-bump solutions for a class of Kirchhoff type problems in $\mathbb{R}^{3}$, J. Math. Phys. 54 (2013), 1-21.

[37] T.F. Ma And J.E. Munoz Rivera, Positive solutions for a nonlinear nonlocal elliptic transmission problem, Appl. Math. Lett. 16 (2003), 243-248.

[38] O. H. Miyagaki, On a class of semilinear elliptic problems in $\mathbb{R}^{N}$ with critical growth, Nonlinear Anal. 29 (1997), 773-781.

[39] J. Moser, A new proof of de Giorgi's theorem concerning the regularity problem for elliptic differential equations, Comm. Pure Appl. Math. 13 (1960), 457-468.

[40] Y.G. Он, On positive multi-bump bound states of nonlinear Schrödinger equations under multiple well potential, Comm. Math. Phys. 131 (1990), 223-253.

[41] K. Perera and Z. Zhang, Nontrivial solutions of Kirchhoff-type problems via the Yang index, J. Differential Equations 221 (2006), 246-255.

[42] E. SÉRÉ, Existence of infinitely many homoclinic orbits in Hamiltonian systems, Math. Z. 209 (1992), 27-42.

[43] F. WANG, On an electromagnetic Schrödinger equation with critical growth, Nonlinear Anal. 69 (2008), 4088-4098.

[44] J. Wang, L. Tian, J. Xu and F. Zhang, Multiplicity and concentration of positive solutions for a Kirchhoff type problem with critical growth, J. Differential Equations 253 (2012), 2314-2351.

[45] M. Willem, Minimax Theorems, Birkhäuser Boston, Boston, MA, 1996. 
[46] J. YAng, Positive solutions of quasilinear elliptic obstacle problems with critical exponents, Nonlinear Anal. 25 (1995), 1283-1306.

Manuscript received December 26, 2014 accepted August 26, 2015

SiHUA LIANG

College of Mathematics

Changchun Normal University

Changchun 130032, Jilin, P.R. CHINA

and

Key Laboratory of Symbolic Computation

and Knowledge Engineering of Ministry of Education

Jilin University

Changchun 130012, P.R. CHINA

E-mail address: liangsihua@163.com

JiHUI ZHANG

Institute of Mathematics

School of Mathematics and Computer Sciences

Nanjing Normal University

210097, Jiangsu, P.R. CHINA

E-mail address: jihuiz@jlonline.com 